\title{
Ion orbits in plasma etching of semiconductors
}

\author{
Tsitsi G. Madziwa-Nussinov ${ }^{\mathrm{a}}$, Donald Arnush ${ }^{\mathrm{b}}$, and Francis F. Chen ${ }^{\mathrm{c}}$ \\ Electrical Engineering Department, University of California, Los Angeles, CA, 90095-1594
}

\begin{abstract}
Fabrication of high-speed semiconductor circuits depends on etching submicron trenches and holes with straight walls, guided by sheath-accelerated ions which strike the substrate at a normal angle. Electrons accumulate at the nonconductive entrance of each trench, charging it negatively and preventing the penetration of electrons to the bottom of the trench. This "electron shading" effect causes an ion charge at the bottom which is well known to cause damage to thin oxide layers. In addition, the deflection of ions by electric fields in the trench can cause deformation of the trench shape. To study this effect, we have calculated the ion orbits self-consistently with their charging of the trench walls. We find that a) the orbits depend only on the electric fields at the entrance and are sensitive to changes in the shape of the photoresist layer there; b) there is an "ion shading” effect that protects part of the wall; and c) the number of ions striking the wall is too small to cause any deformation thereof.
\end{abstract}




\section{INTRODUCTION}

In plasma processing, a planar sheath separates the plasma from the silicon wafer onto which submicron circuits are built by deposition and etching. These features have a minimum size (or critical dimension CD) of $60 \mathrm{~nm}$ or below. The sheath thickness $s$ is at least $5 \lambda_{\mathrm{D}}$, where $\lambda_{D}$ is the Debye length defined below, and can be several times this if a large negative dc bias is applied to the wafer. Taking $n=10^{12} \mathrm{~cm}^{-3}$ and $K T_{\mathrm{e}}=1 \mathrm{eV}$ as extreme values likely to exist at the sheath edge, we find that $s$ is at least $37 \mu \mathrm{m}$, extending over the $200-300 \mathrm{~mm}$ diameter of the wafer. Thus, the sheath is at least 100 times thicker than the feature sizes and orders of magnitude wider. We can therefore assume that ions emitted from the sheath edge have straight trajectories normal to the wafer as they approach its surface.

The phenomenon of electron shading as hypothesized by Hashimoto ${ }^{1}$ is illustrated in Fig. 1. Ions are launched from the sheath edge and are accelerated unidirectionally toward the wafer by the sheath electric field, reaching energies of tens of volts or more. Bombardment of the trench bottom loosens the first few monolayers, allowing the etchant atoms of $\mathrm{Cl}$ or $\mathrm{F}$ to combine much more effectively than without the ions. Electrons, the repelled species, remain in an isotropic Maxwellian distribution and charge the non-conductive photoresist to its floating potential. This negative charge allows very few electrons to enter the trench; thus, the ions deposit a positive charge onto the trench bottom. If the trench is in an insulator, as in this example, no harm is done; but in the over-etch period, the trench can break through into the conducting silicon layer. If this layer is connected to the gate of a transistor, as illustrated at the right, this positive charge can cause a large electric field inside the gate oxide insulator, damaging it. For an oxide thickness of 20A, say, even a 1-V potential drop across it amounts to an electric field of $5 \mathrm{MV} / \mathrm{cm}$. This is a serious problem in computer chip manufacturing, is well known, and is well documented.

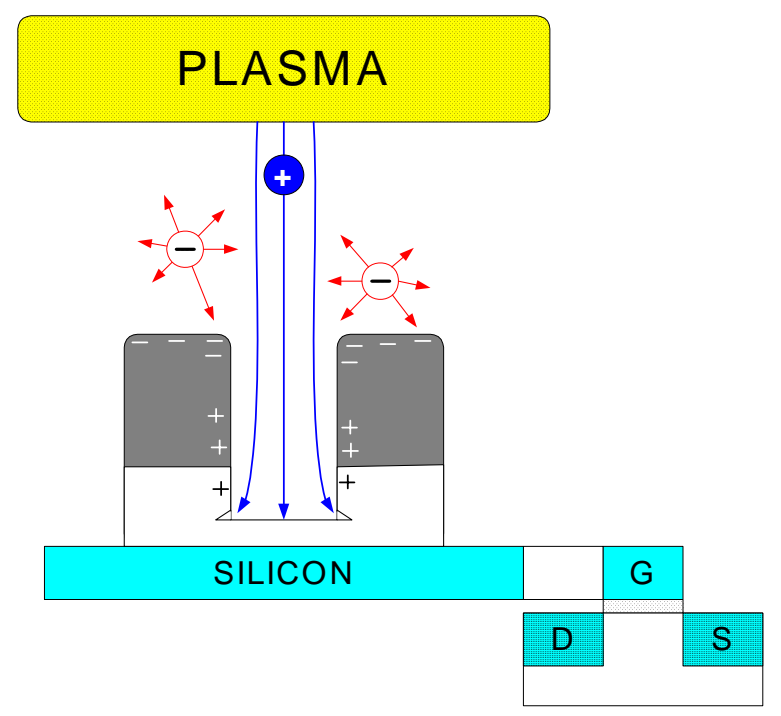

Fig. 1. Geometry of plasma etching of a submicron feature. The dark material at the top is a patterned photoresist mask which defines the trench or via (hole) to be etched. The proximity of the plasma's sheath edge is greatly exaggerated.

In addition, the charging of the walls of a trench, if they are non-conducting, causes electric fields which can deflect the ions. This effect has been cited ${ }^{2}$ as a cause for "notching" and "trenching" deformities observed at trench bottoms. This is also shown in Fig. 1. In the 
present work we try to evaluate this effect quantitatively by calculating the ion orbits selfconsistently with the wall charges which they themselves deposit.

Scale invariance. If they are small enough, the exact size of the trenches is irrelevant; and a universal result can be obtained. To show this, we write Poisson's equation as

$$
\varepsilon_{0} \widehat{\nabla}^{2} V=e\left(n_{e}-n_{i}\right)
$$

where $\hat{V}$ is in dimensional units. In steady state, Eq. (1) determines the electric fields felt by the ions. With the usual definitions

$$
\eta \equiv e \hat{V} / K T_{e}, \quad \lambda_{D}^{2} \equiv \varepsilon_{0} K T_{e} / n_{0} e^{2}, \quad c_{s}^{2} \equiv K T_{e} / M
$$

where $n_{0}$ is the quasineutral plasma density in the main plasma, Eq. (1) can be written

$$
\frac{\varepsilon_{0} K T_{e}}{n_{0} e^{2}} \bar{\nabla}^{2} \eta=\lambda_{D}^{2} \widehat{\nabla}^{2} \eta=\left(\frac{n_{e}}{n_{0}}-\frac{n_{i}}{n_{0}}\right) .
$$

Let $w$ be the scale length of the gradient $\widehat{\nabla}$, and define $\mathbf{r} \equiv \widehat{\mathbf{r}} / w$, so that $\nabla^{2}=w^{2} \widehat{\nabla}^{2}$, yielding

$$
\nabla^{2} \eta=\frac{w^{2}}{\lambda_{D}^{2}}\left(\frac{n_{e}}{n_{0}}-\frac{n_{i}}{n_{0}}\right) \approx 0
$$

We have already shown that $w / \lambda_{\mathrm{D}}$ is extremely small, and it appears squared here. Though $n_{\mathrm{i}}>>$ $n_{\mathrm{e}}$ in the trench, $n_{\mathrm{i}} / n_{0}$ is still $<1$. Thus, as long as $w^{2} / \lambda_{\mathrm{D}}{ }^{2}<<1$, we need only solve the dimensionless Laplace's equation

$$
\nabla^{2} \eta=0
$$

subject to the boundary conditions $\eta=\eta_{b}\left(\mathbf{r}_{b}\right)$ at $\mathbf{r}=\mathbf{r}_{b}$. The solution would be the same as that of the dimensional problem $\widehat{\nabla}^{2} \eta=0$ with the boundary conditions $\eta=\eta_{b}\left(\widehat{\mathbf{r}}_{b}\right)$ at $\widehat{\mathbf{r}}=\widehat{\mathbf{r}}_{b}$. Thus, only the aspect ratio of the trench matters, and not its absolute size, if the Debye length $\lambda_{D}$ is $>>$ $w$. Experiments scaled to macroscopic dimensions can be done as long as this condition is satisfied by going to very low density. The space charge deep inside the sheath is negligible.

The ion trajectories are computed from

$$
\frac{d^{2} \widehat{\mathbf{r}}}{d t^{2}}=-\frac{e}{M} \hat{\nabla} V=-\frac{e}{M} \frac{K T_{e}}{e} \nabla \eta=-c_{s}^{2} \hat{\nabla} \eta .
$$

In terms of $\mathbf{r}$, this becomes $w^{2} d^{2} \mathbf{r} / d t^{2}=-c_{s}^{2} \nabla \eta$. Defining $\tau \equiv c_{s} t / w$, we have

$$
d^{2} \mathbf{r} / d \tau^{2}=-\nabla \eta
$$

which has the same form as Eq. (6), regardless of $w$. Thus, the ion orbits are geometrically the same on any scale; only the time scale is changed. The computations are in these scaleindependent dimensionless units. Collisions are completely negligible, since all mean free paths are longer than $\lambda_{\mathrm{D}}$ and hence much larger than $s$. 


\section{COMPUTATIONAL METHOD}

The two-dimensional region used for computations is shown in Fig. 2. A block of dielectric with $\kappa \approx 4$ is surrounded by a vacuum sheath region bounded by a conductor representing the sheath edge, $S$ dimensionless units away. In practice $S$ is much larger than the feature size and its value is not significant. At the bottom of the trench, shown at the top in this inverted diagram, is a conducting "collector" representing the substrate being etched; the trench grows in the direction of increasing $y$. Ions are accelerated toward the dielectric block by the sheath electric field, and the surface they strike first is normally photoresist. The trench walls are photoresist near the entrance and either oxide or polymer-coated silicon further down. This geometry can represent either polysilicon etch with polymer deposition or oxide etch at the beginning of over-etch. The photoresist surface is divided into cells $x_{\mathrm{j}}$, while the trench walls are divided into smaller cells $y_{j}$. The dielectric has width $2 \mathrm{~L}=14$ and height $\mathrm{H}=10$, while the trench has width $2 \mathrm{~W}$ and depth $\mathrm{D}$, with aspect ratio $\mathrm{A}_{\mathrm{R}}=\mathrm{D} / \mathrm{W}$. Ions are injected vertically from the $V \equiv 0$ sheath edge at $y=0$ with the Bohm velocity $c_{\mathrm{s}}$. The collector is at an adjustable potential $V_{\mathrm{c}}$.

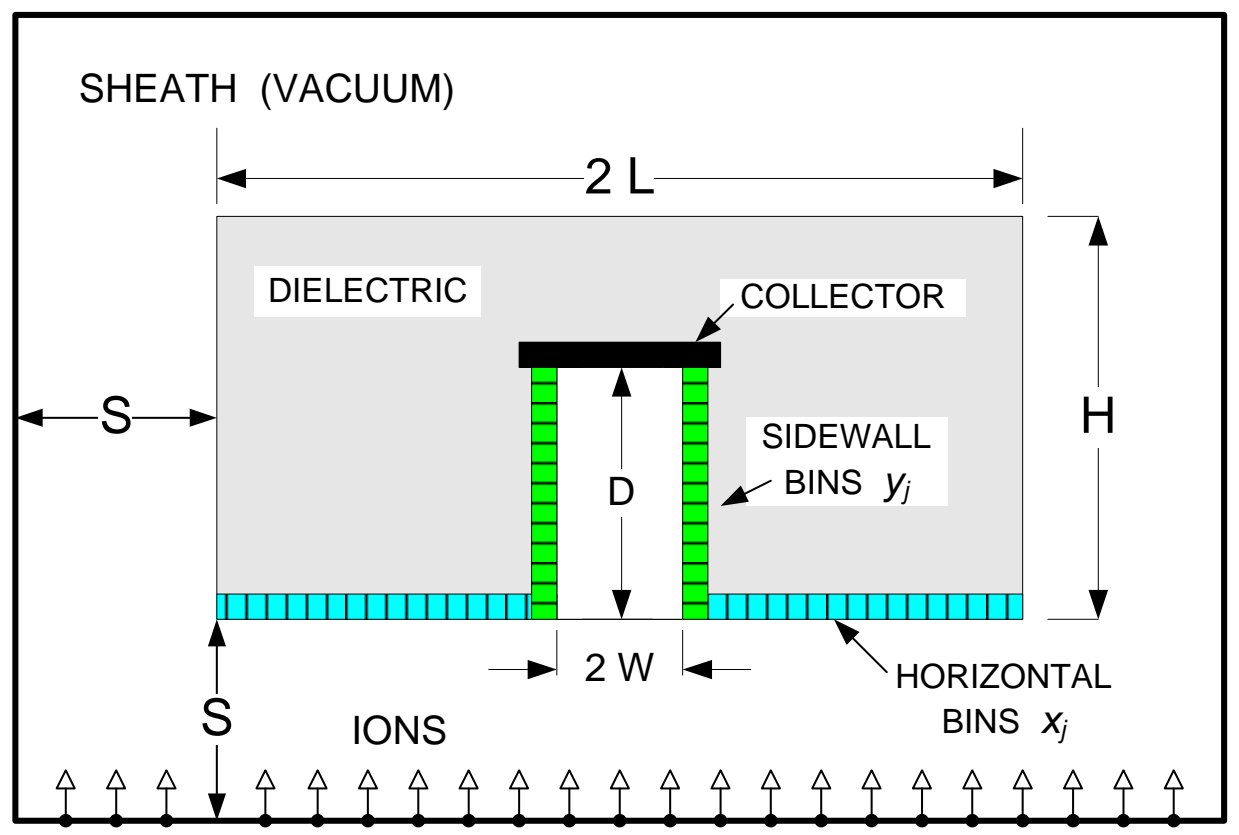

Fig. 2. Computational region. The sheath edge is at the bottom, and the trench is shown inverted, with the collector at the top. The outer region is vacuum, and the inner region a dielectric material. Ions are emitted from the sheath edge at the bottom.

We make three physically reasonable simplifying assumptions: 1) the sheath edge is planar, and monoenergetic ions are ejected at $90^{\circ}$ to it; 2) the ion velocity at the sheath edge has the Bohm value $c_{\mathrm{s}}$, corresponding to an energy $1 / 2 K T_{\mathrm{e}}$; and 3 ) the electrons have a Maxwellian distribution everywhere. The last is true if $V_{\mathrm{c}}$ is negative, as is normal for a biased wafer, so that electrons see a repelling potential everywhere. The Boltzmann relation

$$
n_{e} / n_{s}=\exp \left[\left(V-V_{s}\right) / T_{e V}\right], \quad T_{e V} \equiv K T_{e} / e
$$

then holds for any shape. Here $n_{\mathrm{s}}=n_{\mathrm{i}}=n_{\mathrm{e}}$ at the sheath edge by definition, and we have taken $V_{\mathrm{s}}$ to be 0 . Thus, the bulk plasma has potential $V=+1 / 2 T_{\mathrm{eV}}$. 
The potential on a floating surface is found by equating the electron and ion fluxes. The electron flux is

$$
\Gamma_{e}=n_{e} v_{r}=n_{s} v_{r} \exp \left[\left(V-V_{s}\right) / T_{e V}\right], \text { where } v_{r}=\left(K T_{e} / 2 \pi m\right)^{1 / 2}
$$

is the random thermal velocity normal to a surface. The ion flux at $y=0$ is simply

$$
\Gamma_{0} \equiv \Gamma_{i}(0)=n_{s} c_{S}=n_{S}\left(K T_{e} / M\right)^{1 / 2} .
$$

In the absence of a trench, the substrate surface at $y=6$ charges to the usual floating potential $V_{\mathrm{f}}$ given by $\Gamma_{\mathrm{i}}(6)=\Gamma_{\mathrm{i}}(0)=\Gamma_{\mathrm{e}}$ :

$$
\left(V_{f}-V_{s}\right) / T_{e V}=-\ln (M / 2 \pi m)^{1 / 2} \approx-4.68 \text { for argon. }
$$

We have set $V_{\mathrm{s}}=0$, so that the computation is in a grounded box. Since $V_{\mathrm{s}}$ is $\approx-1 / 2 T_{e V}$ relative to the plasma, $V_{\mathrm{f}}$ is $\approx-5.18 T_{\mathrm{eV}}$ relative to the plasma or $\approx-15 \mathrm{~V}$ for $K T_{\mathrm{e}}=3 \mathrm{eV}$ in argon.

The potential to which each surface element $\Delta x_{\mathrm{j}}$ of the photoresist or $\Delta \mathrm{y}_{\mathrm{j}}$ of the trench wall charges is computed as follows. Let $N$ be the number of ions $\left(\approx 10^{4}\right)$ emitted at $y=0$ over a surface area $\mathrm{LZ}$ per unit time, where $\mathrm{Z}$ is a length in the ignorable $\mathrm{Z}$ direction. The emitted ion flux is $\Gamma_{0}=N / L Z=n_{\mathrm{s}} C_{\mathrm{s}}$. If $N_{\mathrm{j}}$ ions strike a surface cell of width $\Delta x_{\mathrm{j}}$, the ion flux to that cell is $\Gamma_{\mathrm{i}, \mathrm{j}}=N_{\mathrm{j}} / \Delta x_{\mathrm{j}} \mathrm{Z}$. The ratio of this to the undisturbed flux $\Gamma_{0}$ is then

$$
R\left(x_{j}\right)=\left(N_{j} / N\right)\left(L / \Delta x_{j}\right)=F\left(x_{j}\right)\left(L / \Delta x_{j}\right),
$$

where $F\left(x_{\mathrm{j}}\right)$ is the fraction of all ions that end up in cell $x_{\mathrm{j}}$. The electron flux $\Gamma_{\mathrm{e}, \mathrm{j}}$ to a cell is $n_{s} v_{r} \exp \left(V_{j} / T_{e V}\right)$. Equating this to the ion flux $\Gamma_{\mathrm{i}, \mathrm{j}}=n_{\mathrm{s}} c_{\mathrm{s}} R\left(x_{\mathrm{j}}\right)$, we have

$$
v_{r} e^{V / T_{e V}}=c_{S} F\left(x_{j}\right) L / \Delta x_{j}
$$

Using Eq. (11), we find the floating potential of that cell relative to the sheath edge to be

$$
V\left(x_{j}\right)=T_{e V}\left[\ln \left(F_{j} L / \Delta x_{j}\right)-4.68\right],
$$

where $F\left(x_{\mathrm{j}}\right)$ is found by counting trajectories.

Fig. 3. Flow diagram for the computation.

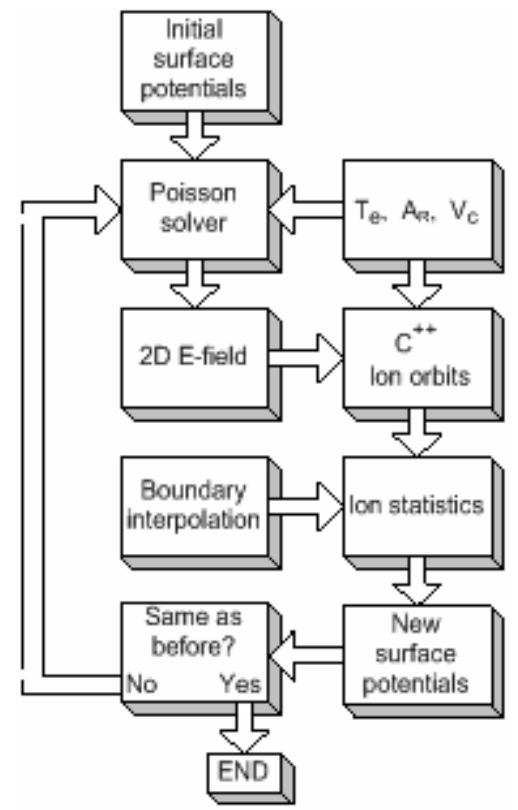


The computational loop is shown in Fig. 3. For a plasma characterized by $K T_{\mathrm{e}}$ and ion charge-to-mass ratio, ion orbits are computed first with all insulating surfaces at potential $V_{\mathrm{f}}$ and the collector at potential $V_{\mathrm{c}}$. A Poisson solver with a triangular grid ${ }^{3}$ is used to calculate the 2D electric fields. The time-independent trajectory of each ion emitted from the sheath edge is then calculated with a $\mathrm{C}++$ program written for this purpose. When the trajectory intersects a surface cell, its contribution to $N_{\mathrm{j}}$ is counted. The potential of each cell $V\left(x_{\mathrm{j}}\right)$ is then calculated from Eq. (14).and used in the first iteration. The ion orbits are then recalculated, giving data for the next iteration. This is continued until $N_{\mathrm{j}}$ and $V\left(x_{\mathrm{j}}\right)$ converge to steady values. When no ion falls on a cell, Eq. (14) diverges. In that case, we assume that the cell actually receives one ion or a fraction of an ion, resulting in $V\left(x_{\mathrm{j}}\right) \approx-40 \mathrm{~V}$. The results are not sensitive to this approximation. In this model, we neglect all collisions, secondary emission, and surface currents, though a test of the last effect will be mentioned later. Details of the method are given in a dissertation ${ }^{4}$.
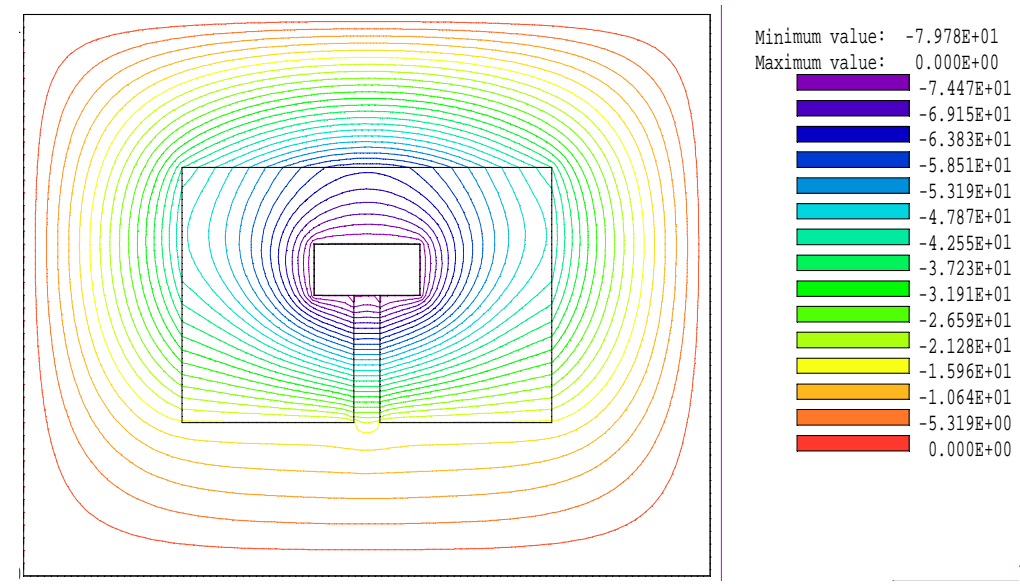

Fig. 4. Vacuum equipotential contours in the computational region for $A_{\mathrm{R}}=4, V_{\mathrm{c}}=-80 \mathrm{~V}$.

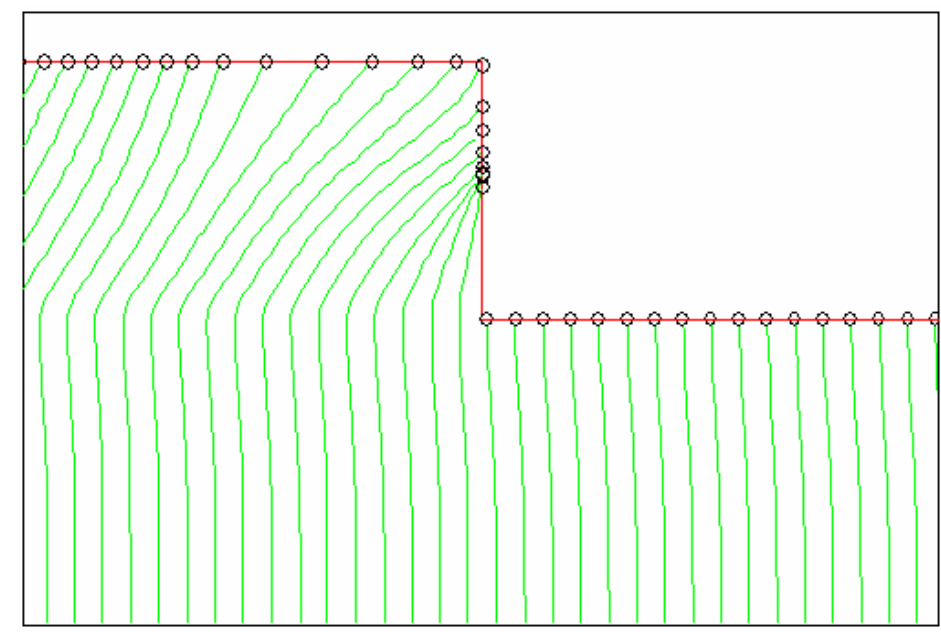

Fig. 5. Example of ion orbit intersections with a boundary. The discrete ion positions usually straddle the boundary, and must be interpolated to get the exact position on the boundary.

Because of the scale invariance of the problem, only two parameters need to be set for any given geometry: the aspect ratio $A_{\mathrm{R}}$ and the collector potential $V_{\mathrm{c}}$. For definiteness, all dielectrics are given a representative value of $\varepsilon_{R}=4$. For example, Fig. 4 shows the vacuum equipotential lines for $A_{\mathrm{R}}=4, V_{\mathrm{c}}=-80 \mathrm{~V}$, and the dielectric surfaces at the initial floating potential of $-4.68 T_{\mathrm{eV}}=14 \mathrm{~V}$ for $K T_{\mathrm{e}}=3 \mathrm{eV}$. Figure 5 shows how the ion charges are counted. 
Since the "time" steps are discrete, an ion trajectory does not necessarily intersect the boundary at the end of a step; the position has to be interpolated. The number of such stopping points in each cell provides the value of $N_{\mathrm{j}}$ described above. Once the iteration has converged, the orbits are universal curves for the given values of $A_{\mathrm{R}}, V_{\mathrm{c}}$, and $K T_{\mathrm{e}}$. The density is irrelevant since, as shown above, the space charge is negligible. The size of the dielectric block does not matter as long as it it big enough. Figure 6 shows the distribution of ions $N_{j}$ to the cells $y_{\mathrm{j}}$ on the trench wall after the first two iterations and the final one. In some cases, $V\left(x_{\mathrm{j}}\right)$ does not converge but oscillates between two or three patterns after 25 iterations. This phenomenon will be discussed in a later section.
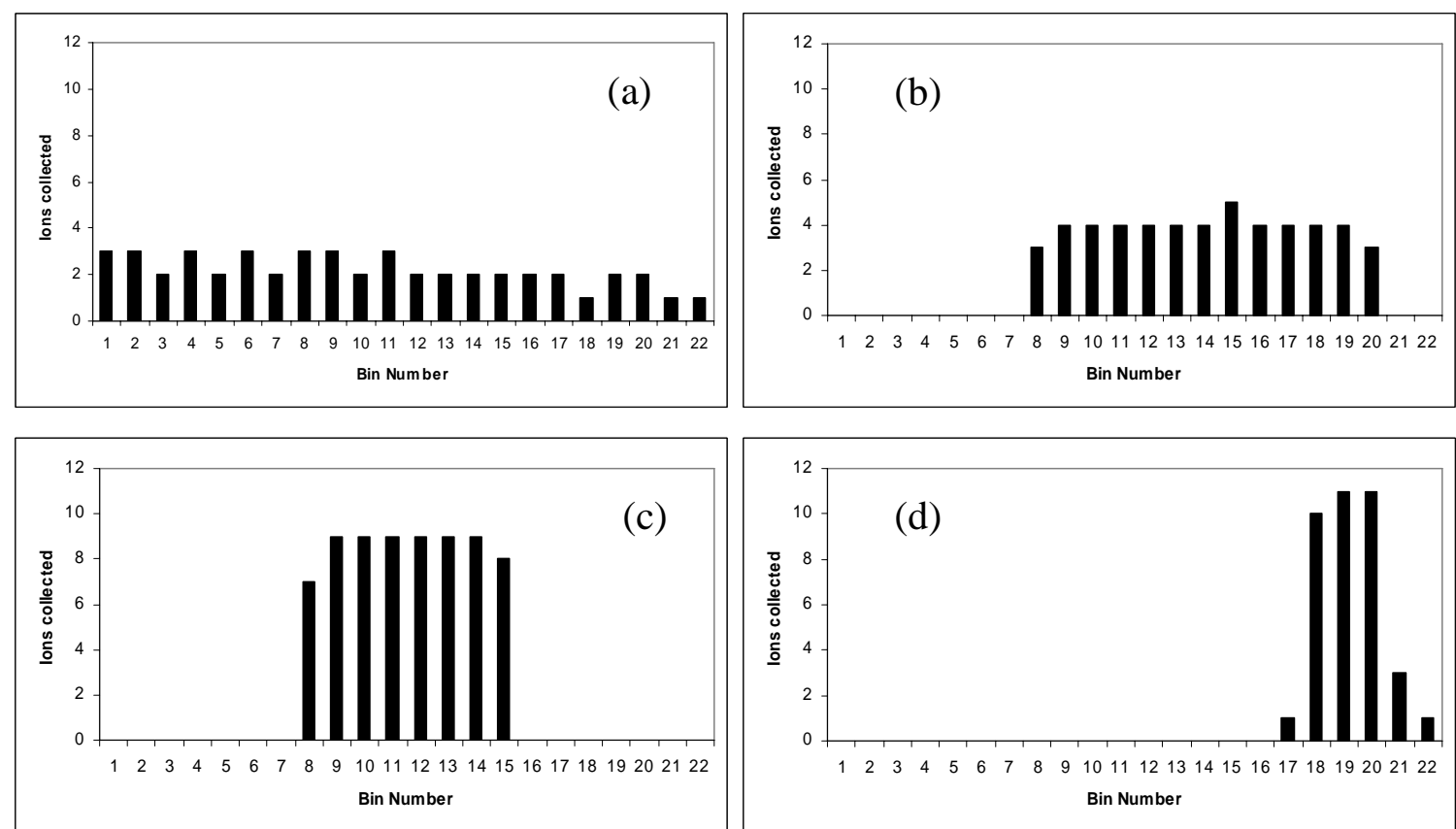

Fig. 6. Evolution of the ion flux to the bins on the trench wall as the fields are iterated. The trench entrance is on the left, and the collector on the right. $A_{\mathrm{R}}=7, V_{\mathrm{c}}=-18 \mathrm{~V}$.

\section{RESULTS}

\section{A. Ion shading}

The E-field pattern near the trench entrance is shown in Fig. 7. Because of the sharp corners, the field is extremely strong there and causes a large deflection of the ions, as shown in Fig. 9a. To suppress this unphysical effect, the corners were subsequently rounded into a circular arc, as shown in Fig. 8. The resulting trajectories (Fig. 9b) are more reasonable, but the fact remains that no ions can strike the sidewall near the entrance. This ion shadowing effect, which occurs in addition to the well-known electron shading effect, is caused by the E-fields outside the trench, which curve the orbits inward. At large negative $V_{\mathrm{c}}$ 's, the entire sidewall can be protected from ion bombardment; this is shown in Fig. 10. The percentage of ions collected on the sidewall as a function of $V_{\mathrm{c}}$ is shown in Fig. 11 for three values of $A_{\mathrm{R}}$. The decrease is more or less exponential. Physically, the ions gain enough energy at large $\left|V_{c}\right|$ that the negative sidewalls cannot deflect them. 


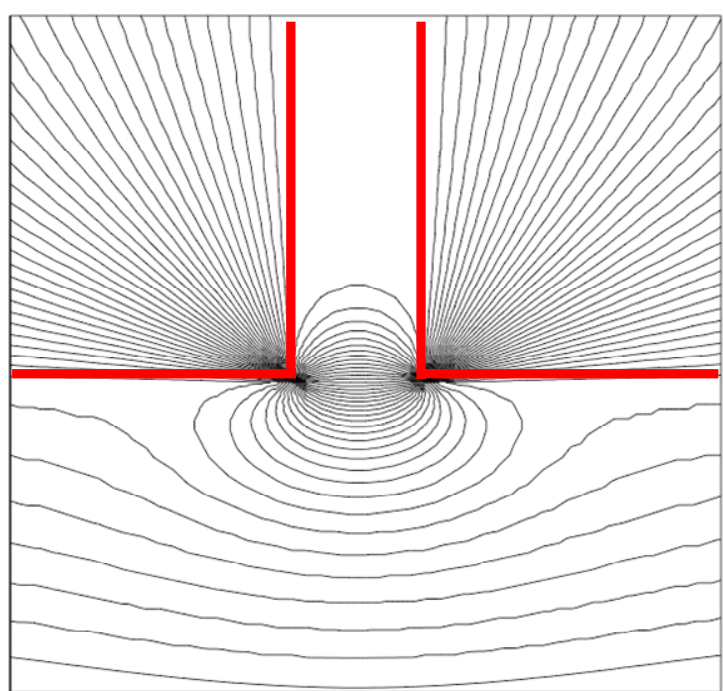

Fig. 7. Equipotentials near a square trench entrance.

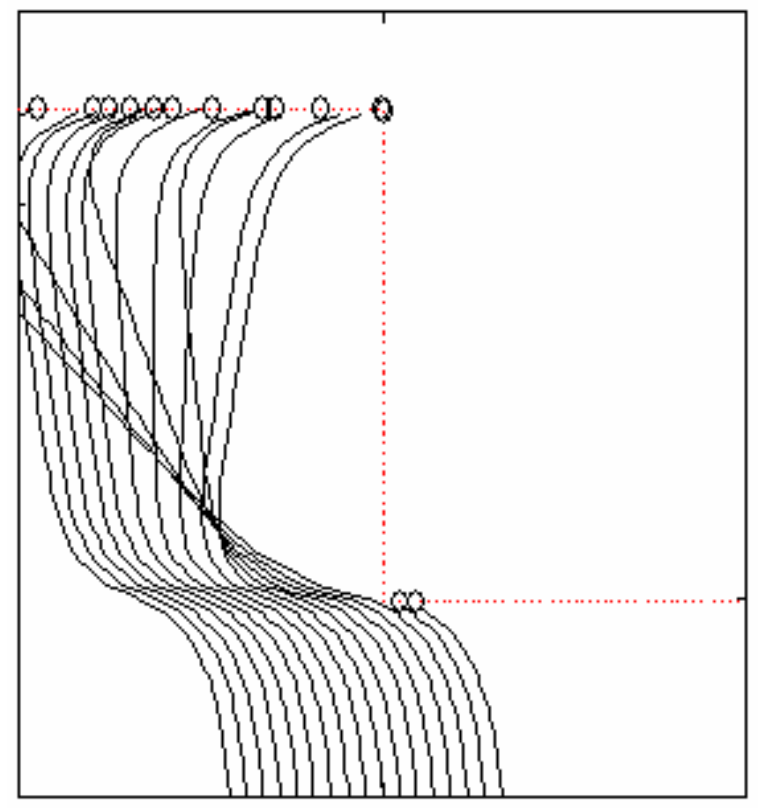

(a)

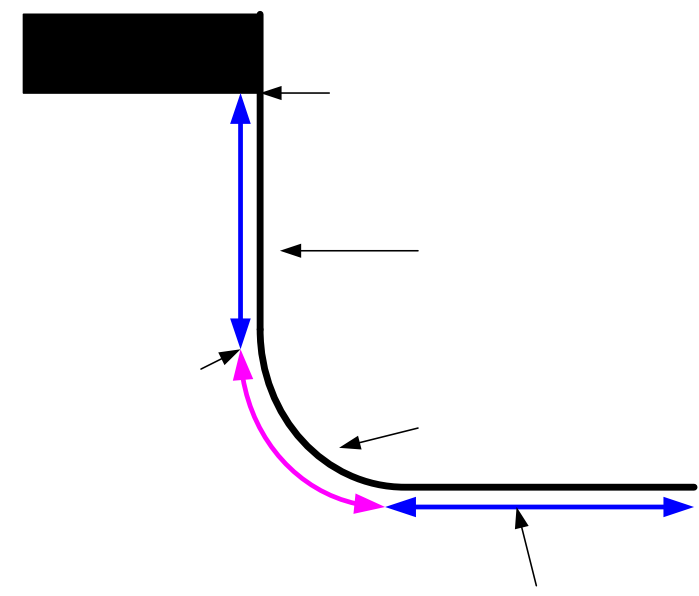

Fig. 8. A rounded trench entrance, showing three regions (dielectric surface, arc, and sidewall), where the bins have different sizes.

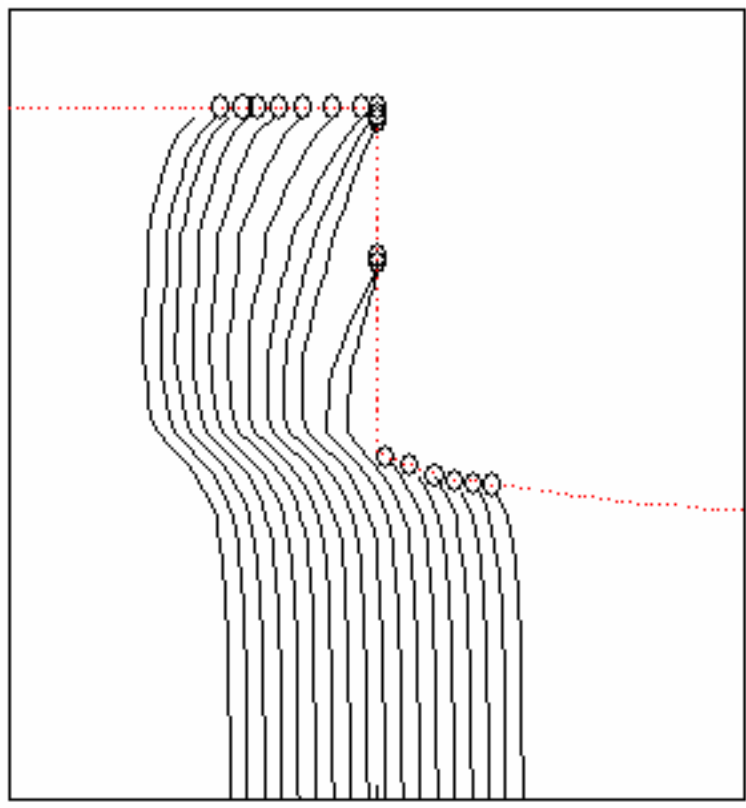

(b)

Fig. 9. Ion orbits a) in the field of Fig. 7 and b) with rounded corners. The horizontal scale has been expanded to show the effect. 


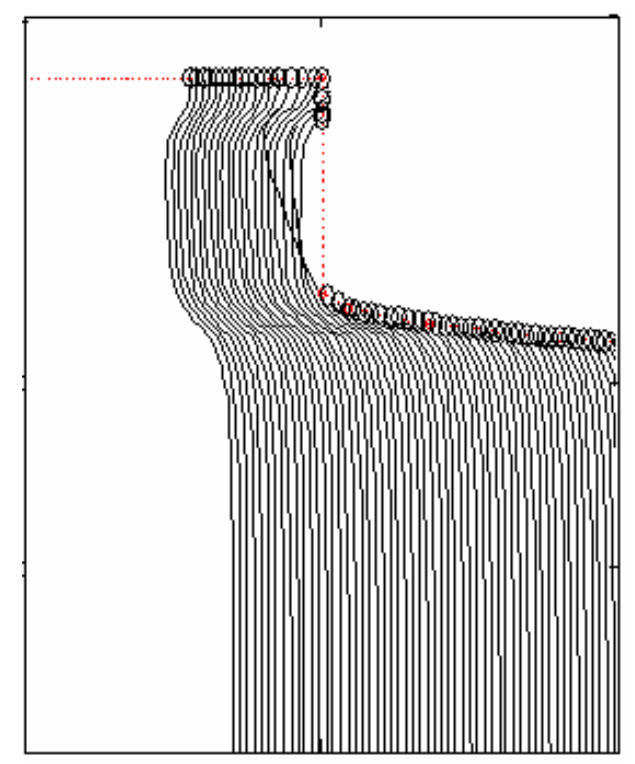

(a)

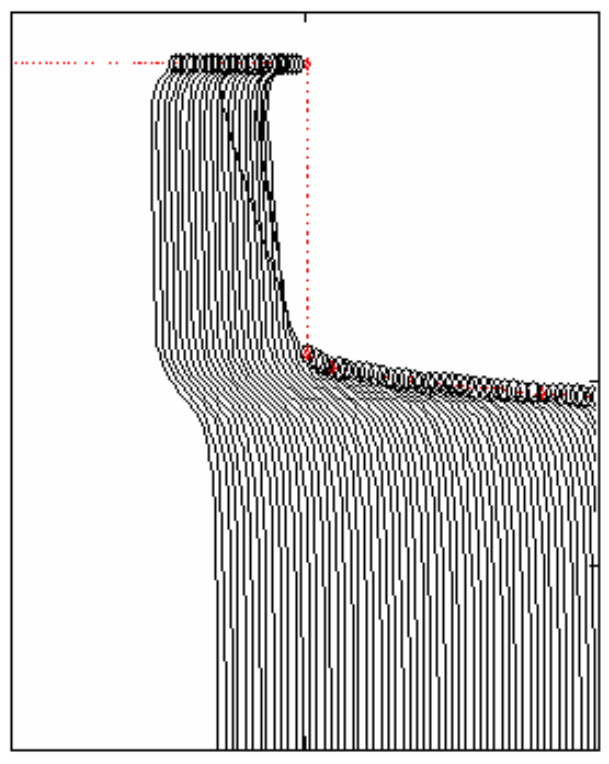

(b)

Fig. 10. Ion shading effect for $\mathrm{AR}=5$ and a) $V_{\mathrm{c}}=26 \mathrm{~V}$ and b) $V_{\mathrm{c}}=40 \mathrm{~V}$. The horizontal scale has been expanded.

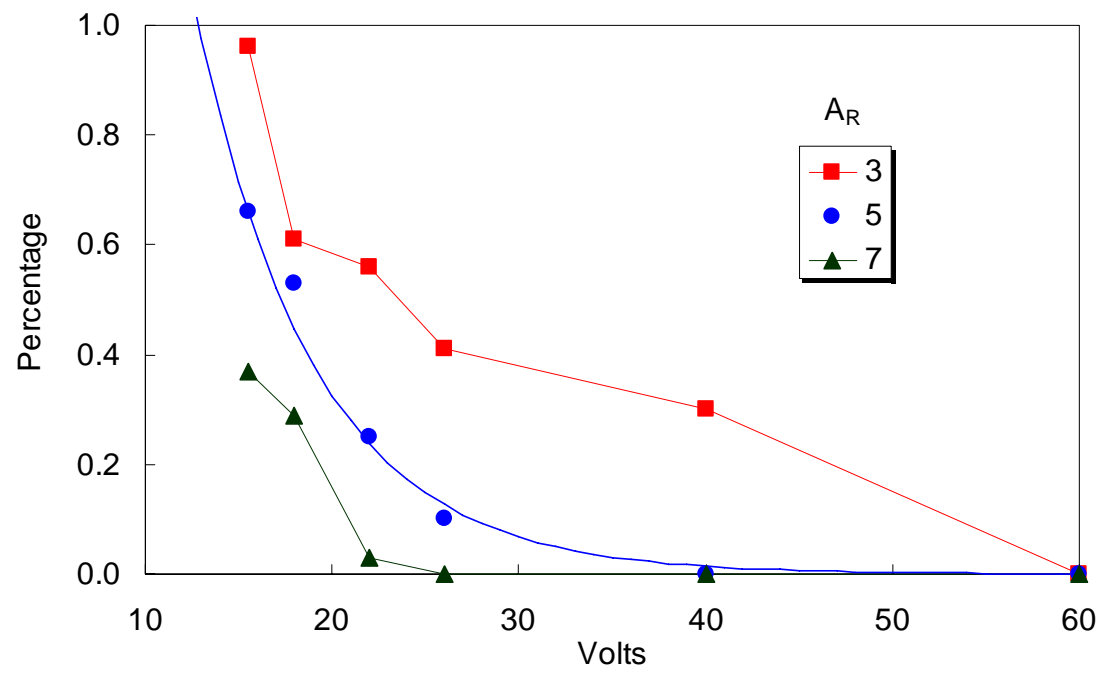

Fig. 11. Percentage of emitted ions falling on the sidewalls as a function of collector bias $\left|V_{\mathrm{c}}\right|$ for three values of $A_{\mathrm{R}}$. The curve is an exponential fit to the $A_{\mathrm{R}}=5$ data.

That the ion shading increases with $A_{\mathrm{R}}$ is a more subtle effect. Figure 12 shows the shaded regions for three values of $A_{\mathrm{R}}$ at constant $V_{\mathrm{c}}$. The reason that ions are deflected into the sidewalls only for low $A_{\mathrm{R}}$ can be seen from the self-consistent field patterns in Fig. 13. It is seen that the fields are very strong at the ends of the trench, particularly at the corners. At $A_{\mathrm{R}}=7$, the interior of the trench is essentially field-free. Ions are deflected into the sidewall only in the arc region. At $A_{\mathrm{R}}=3$, the fields extend into the interior, where $E_{\mathrm{x}}$ is large enough to impart transverse momentum to the ions streaking in the $y$ direction.

Ion shading causes the collector current to saturate above a critical $V_{\mathrm{c}}$ above which no ions can be lost to the sidewalls. This is shown in Fig. 14. The decrease for $\left|V_{\mathrm{c}}\right|$ less than about $20 \mathrm{~V}$ is the loss to the sidewalls in Fig. 11, plus the loss to the arc region. On the right-hand scale 
is shown the percentage of ions emitted in the area geometrically over the trench. This exceeds $100 \%$ because ions are drawn into the trench by external E-fields.
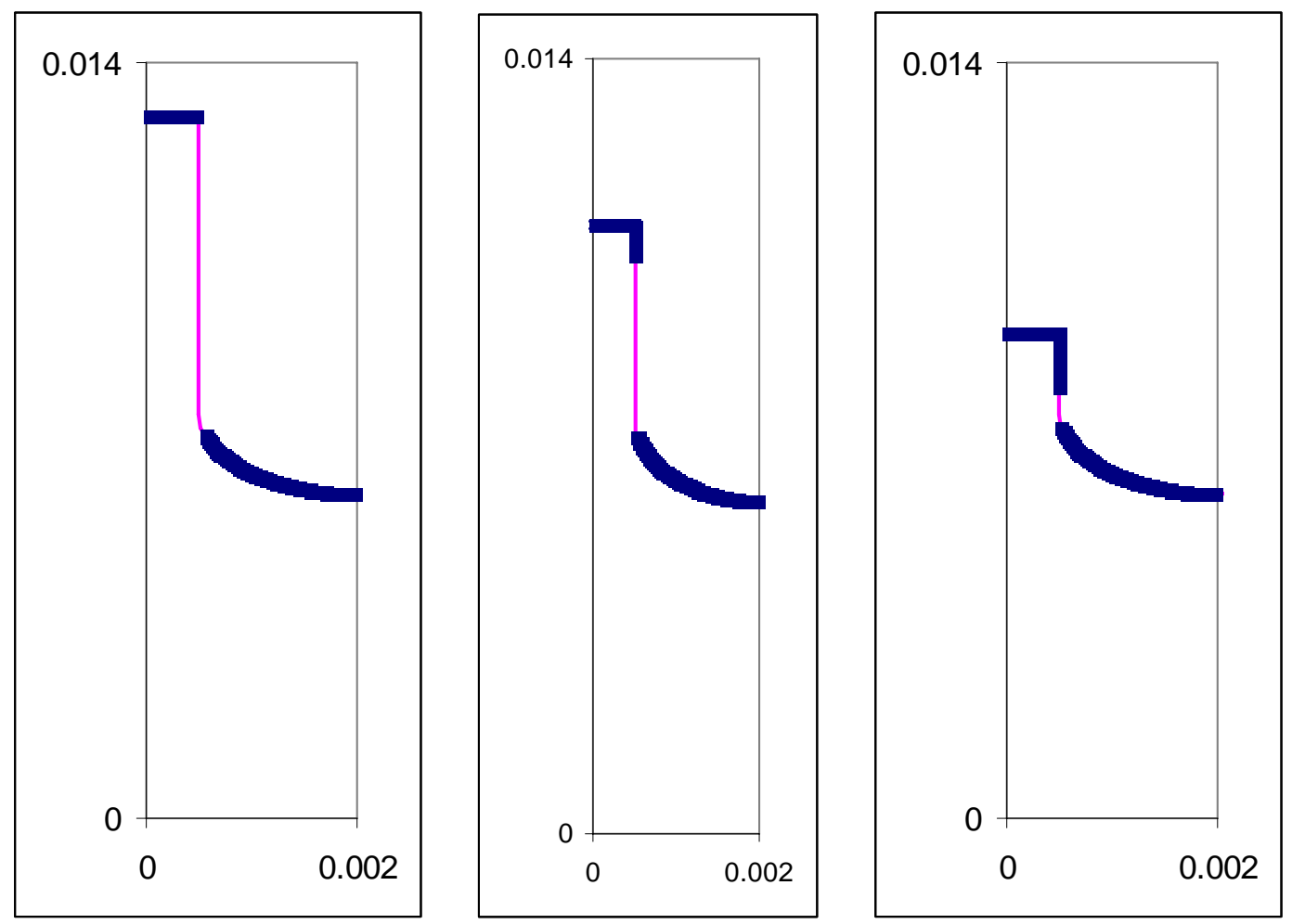

Fig. 12. Ion collection regions in trenches with $V_{c}=-26 \mathrm{~V}$ and $A_{R}=7,5$, and 3, respectively. The light regions of the trench wall are not struck by any ions.
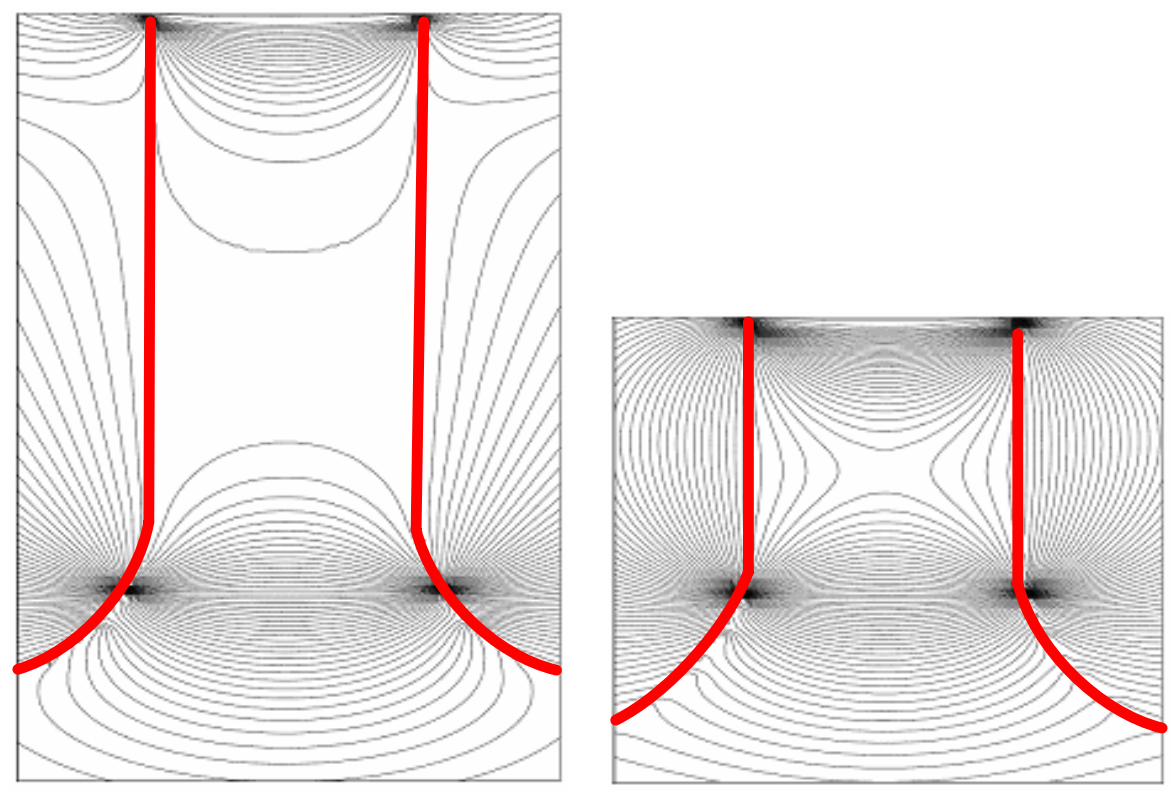

Fig. 13. E-field patterns (equipotentials) for $A_{\mathrm{R}}=7$ and 3, at $V_{\mathrm{c}}=-22 \mathrm{~V}$. 


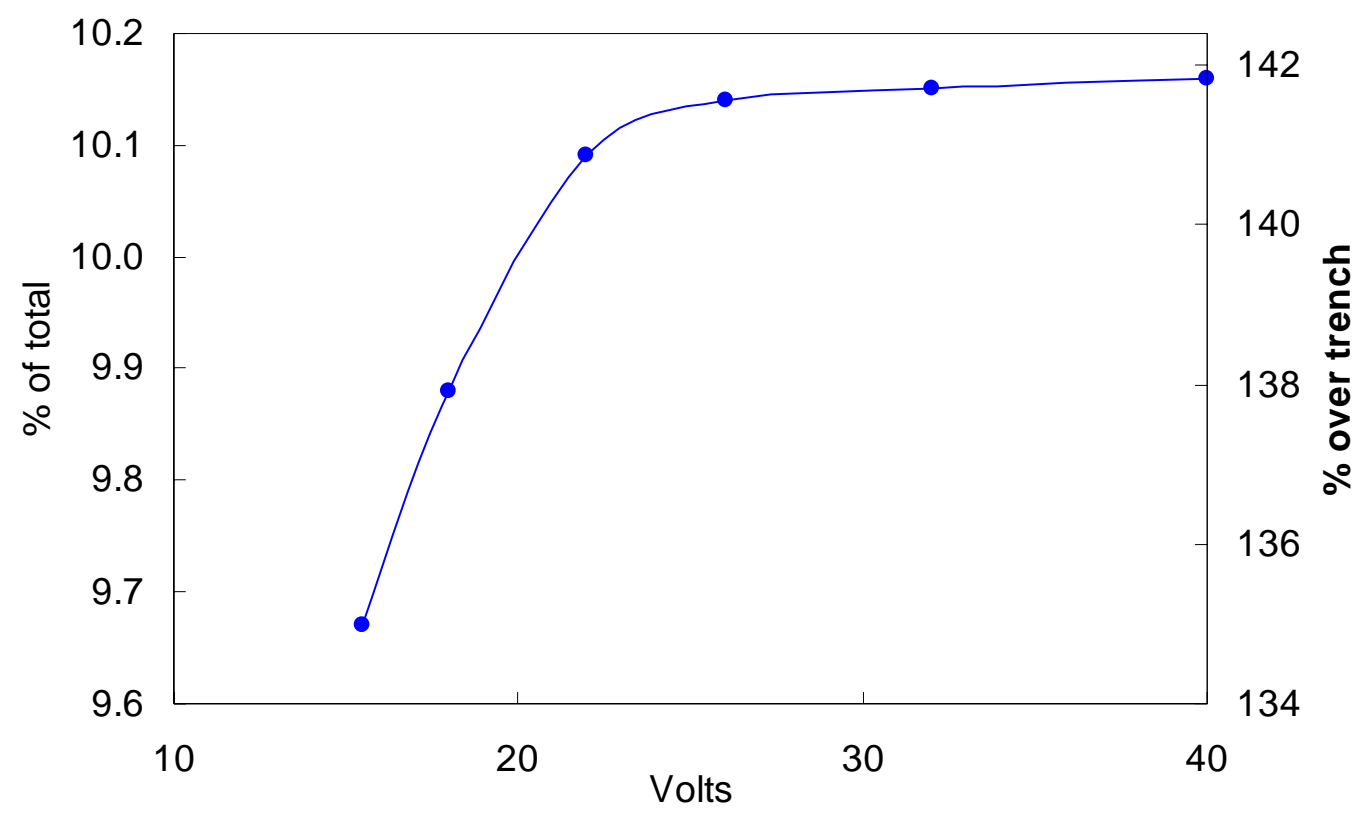

Fig. 14. Number of ions falling on the collector at $A_{\mathrm{R}}=7$ as a function of collector voltage, expressed as a percentage of all ions emitted from $x=-L$ to $L$ (left scale) and as a percentage of ions emitted over the trench area (right scale).

\section{B. Nature of the ion trajectories}

The quantitative results can be better understood if one first observes the nature of the ion orbits. Figure 15 shows three views of the trajectories at $A_{\mathrm{R}}=7$ and $V_{\mathrm{c}}=-26 \mathrm{~V}$. This figure has the normal orientation, with the substrate at the bottom, and is inverted relative to the computational grid of Fig. 2. Figure 15a shows the orbits on a normal $x-y$ scale. The ions enter the trench at high velocity, and their deflections are so small that they cannot be seen on this scale. In Fig. 15b, the horizontal scale has been expanded by a factor 20 . Here it can be seen that the ions enter the trench at an angle due to the external E-field. No ions strike the first part of the wall, which is shaded. The negative charges on the wall eventually deflect the ions outward, causing them to strike the corner of the trench. In Fig. 15c, the horizontal scale has been stretched by a factor 80 , and intermediate orbits have been plotted, shown by the thin lines. Orbits which pass near the corner of the photoresist actually cross and take shapes that are sensitive to the strong fields at the entrance corner. Note that the corner is actually circular but looks sharp only on this 80-to- 1 scale. The pulling of ions into the trench by the charge on the photoresist is shown more clearly in Fig. 16. Incident ions (indicated by the solid lines) headed for the photoresist surface are drawn into the trench by the fields in the arc region. Since this region is charged negatively, the deflection direction is counter-intuitive. However, the equipotentials shown in Fig. 13 show that the net E-field from all negative surfaces actually point away from the arc region. 


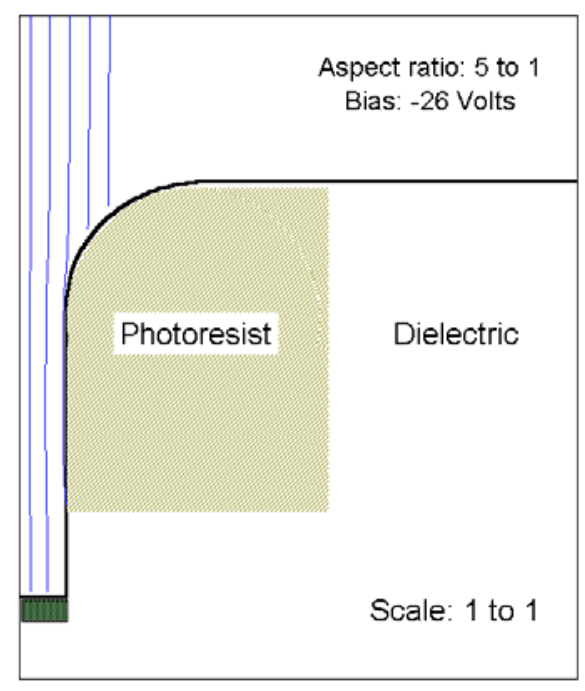

Fig. 15(a)

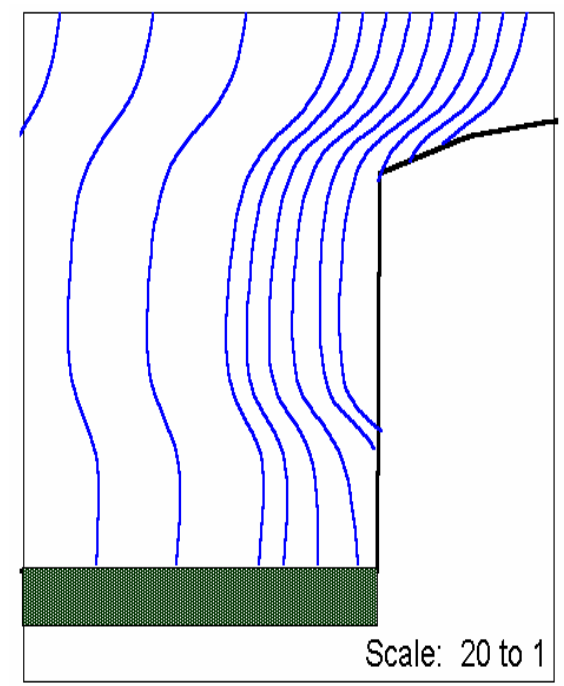

Fig. 15(b)

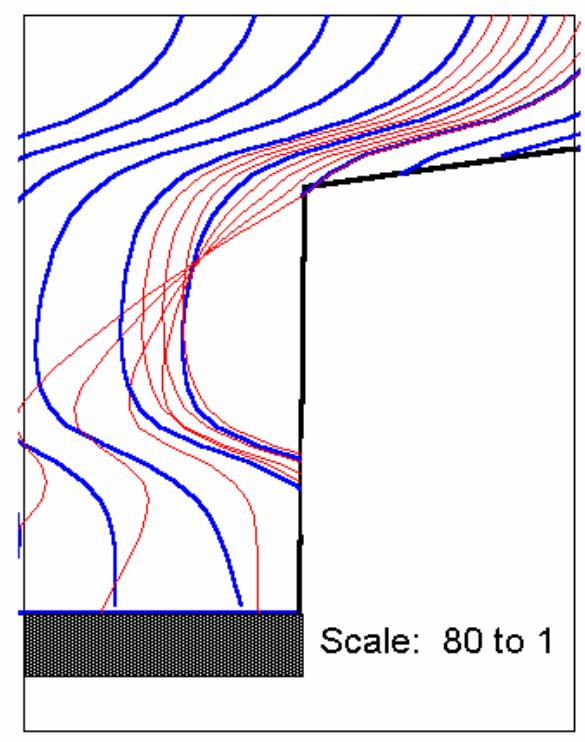

Fig. 15(c)

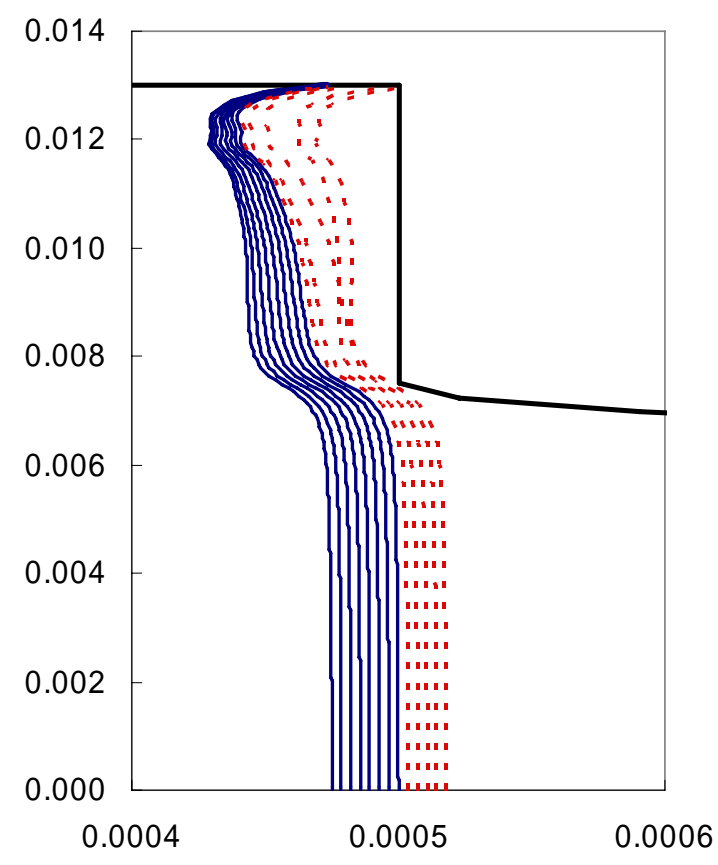

Fig. 16

Fig. 15. Ion orbits for $A_{\mathrm{R}}=5$ and $\left|V_{\mathrm{c}}\right|=26 \mathrm{~V}$ with the horizontal scale (a) normal, (b) expanded by a factor 20, and (c) expanded by a factor 80 relative to the vertical scale. The orbit spacing has been decreased near the wall for clarity. Fig. 16. Ion orbits near the trench edge for $A_{\mathrm{R}}=7$ and $V_{\mathrm{c}}=22$. The figure is inverted relative to Fig. 15 .

The ions also arrive at the collector with different energies. Figure 17 shows the ion perpendicular velocities as they strike various parts of the collector. Those near the sidewall are moving laterally and therefore would not penetrate as deeply into the surface of the collector. The curve is double-valued: the low-energy ions are those in crossing orbits (Fig. 15c).

Fig. 17 


\section{Effect of photoresist shape}

As etching progresses, the photoresist will change its shape at the trench entrance, and this will affect the ion orbits. Since the E-fields are strongest there, we expect that the ion orbits would be sensitive to these changes. First, consider the change from a mathematically sharp edge to the rounded edge used in our calculations. This was originally done to improve the convergence of the iterations, but the change in orbits was significant. Table 1 shows the percentage of ions landing on each part of the trench for a sample of about $10^{4}$ ions emitted from the entire sheath area. On the photoresist surface, including the arc region, the potential is solved self-consistently, as it was on the trench walls.

Table 1: Ion distributions with sharp and rounded corners

$\begin{array}{rcc}\boldsymbol{A}_{\mathbf{R}}=\mathbf{7}, \boldsymbol{V}_{\mathbf{c}}=\mathbf{- 1 8 V} & \begin{array}{c}\text { Case A: } \\ \text { Sharp corner }\end{array} & \begin{array}{c}\text { Case } \mathbf{B}: \\ \text { Rounded corner }\end{array} \\ \text { Collector } & 7.9 \% & 9.9 \% \\ \text { Sidewalls } & 0.5 \% & 0.3 \% \\ \text { Arc } & \text { N/A } & 20.7 \% \\ \text { Flat surface } & 91.6 \% & 69.1 \% \\ & & \\ \boldsymbol{A}_{\mathbf{R}}=\mathbf{7}, \boldsymbol{V}_{\mathbf{c}}=-\mathbf{2 6 V} & & \\ \text { Collector } & 8.2 \% & 10.1 \% \\ \text { Sidewalls } & 0.18 \% & 0.00 \%\end{array}$

Table 1 shows that fewer ions reach the collector if the corner is sharp rather than rounded. This is because the sharp corner more effectively shields ions approaching the trench at an angle. In either case, the fraction of ions hitting the sidewall is extremely small and cannot cause the trench profile defects that have been observed. As expected, the collector current increases and sidewall current decreases at the higher $\left|V_{\mathrm{c}}\right|$ of $26 \mathrm{~V}$. The difference between cases $\mathrm{A}$ and $\mathrm{B}$ is even more noticeable in the distribution of ions shown in Fig. 18. In case A, ions are collected over most of the sidewall, while in case B ions are shielded from all but the last bin.

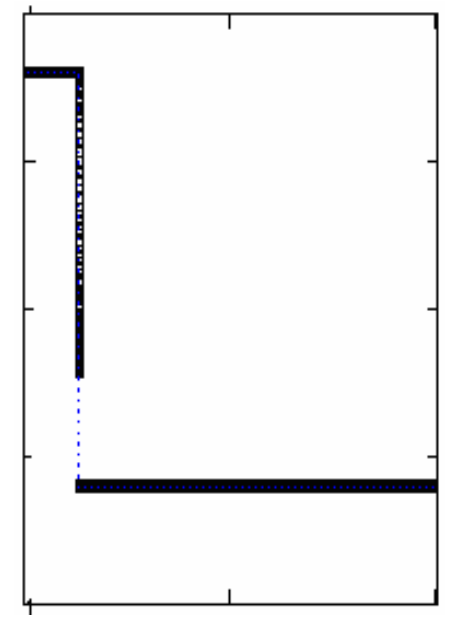

(a)

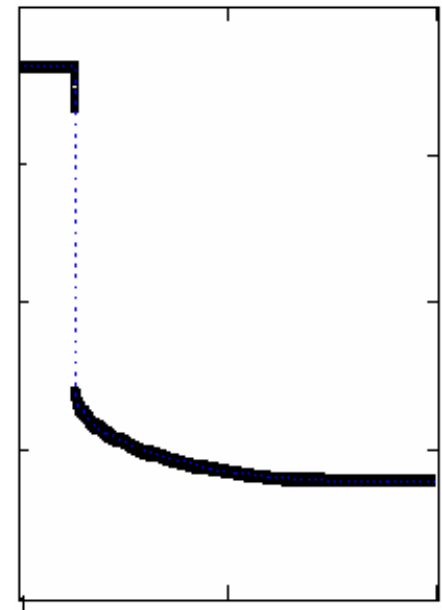

(b) 


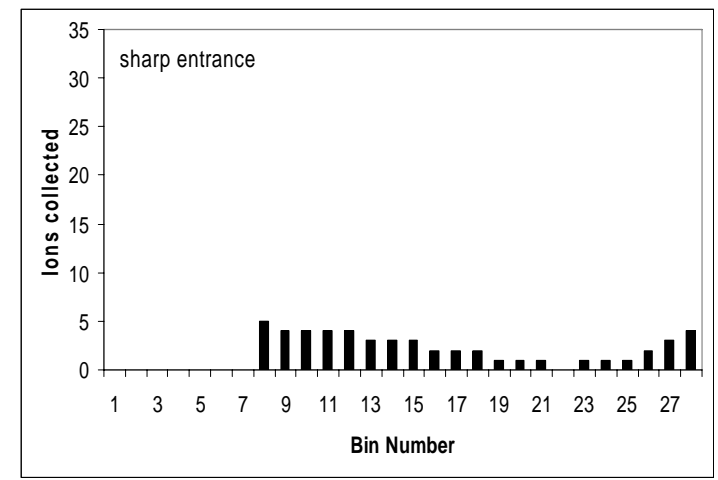

(c)

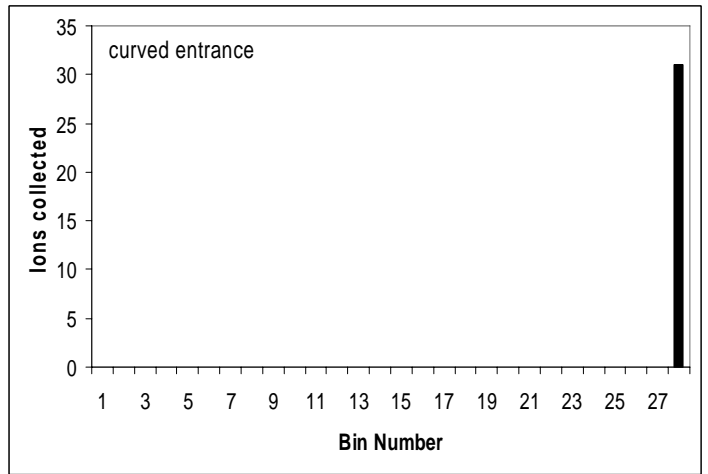

(d)

Fig. 18. Distribution of ion collection for sharp (a and c) and rounded (b and d) trench entrances, as shown on an $x-y$ plot (a and b) and as a histogram of the sidewall bins (c and d).
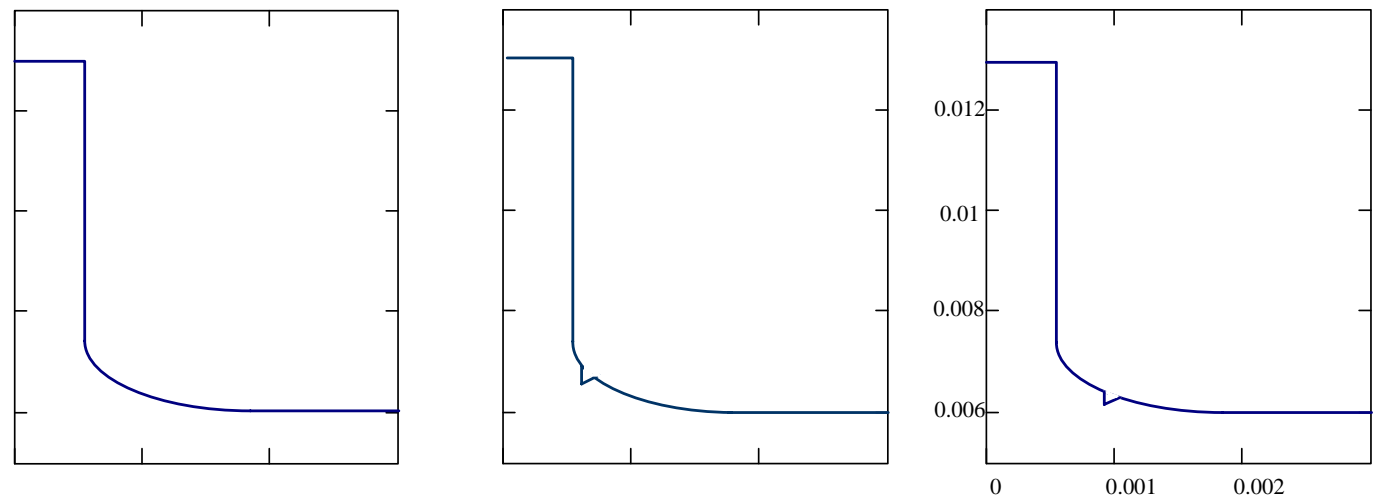

Fig. 19. Profiles of trench entrances without and with bumps.

To see the effect of small changes in the entrance shape, we added a small bump onto the arc region in two locations. The statistics are shown in Table 2 for a sample of 50,000 ions. In case D, the bump is farther from the trench than in case C (Fig. 19). The sidewall distributions

Table 2: Ion distributions with small bumps

$\begin{array}{rrcc}\boldsymbol{A}_{\mathbf{R}}=\mathbf{7}, \boldsymbol{V}_{\mathbf{c}}=\mathbf{- 1 8 V} & \text { Case B: No bump } & \text { Case C: Near bump } & \text { Case D: Far bump } \\ \text { Collector } & 9.9 \% & 8.6 \% & 9.0 \% \\ \text { Sidewalls } & 0.0120 .3 \% & 0.0 \% & 1.8 \% 0.012 \\ \text { Arc } & 20.7 \% & 17.7 \% & 17.4 \% \\ \text { Flat surface } & 69.1 \% & 73.7 \% & 71.8 \% \\ & 0.01 & & 0.01\end{array}$

are shown in Fig. 20. It is clear that even a small deformation of the photoresist will change the ion orbits drastically. The sidewall ions then cause fields within the trench. The self-consistent equipotential lines for these thefe cases are shown in Fig. 21. In case B, with a roundeg grtrance but no bumps, the E-field is very strong near the entrance. The ions are given a kick there and then coast to the trench bottom (at the top in the figure) through the nearly field-free trench. The 
Fig. 20
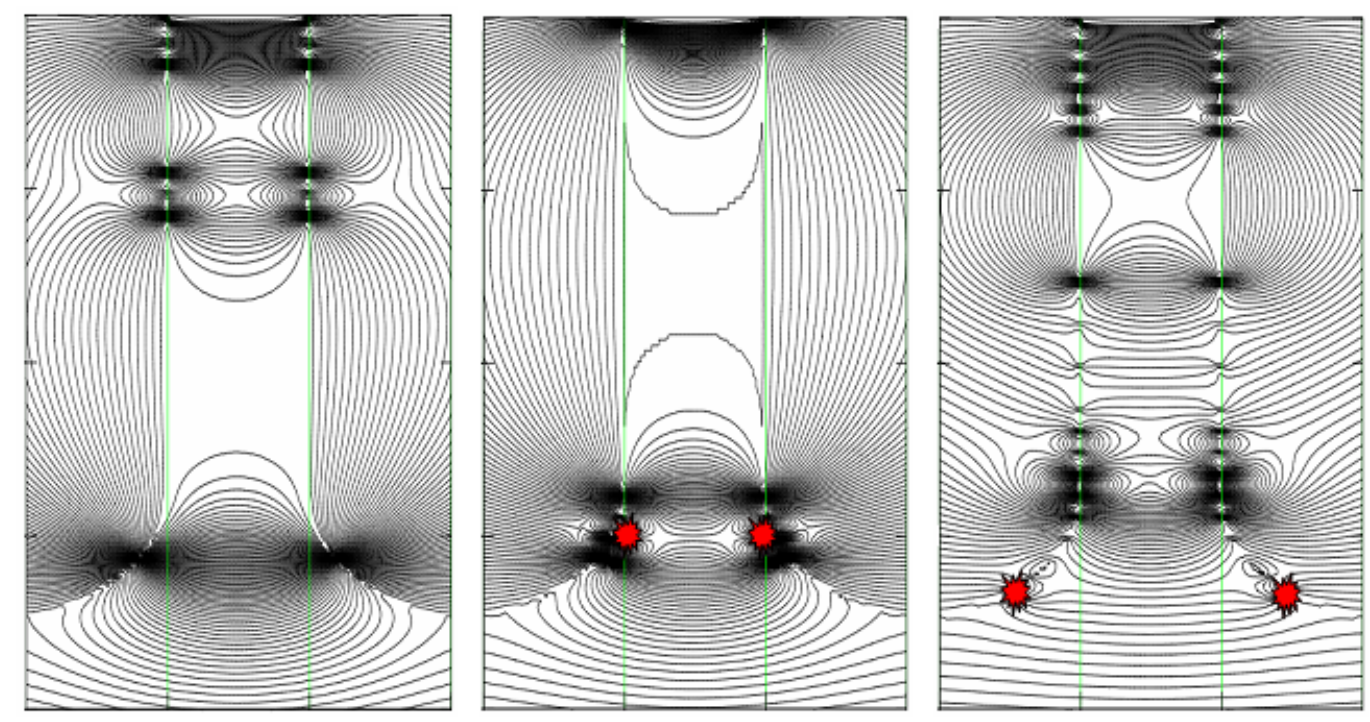

Fig. 21. E-field patterns for case B (no bumps), (C) near bumps, and (D) far bumps on the photoresist. The position of the bumps is indicated by the red dots in the color version.

collector is given only a small bias in this example, so that ions are not strongly drawn into it; some of them hit the sidewall near the bottom, giving rise to E-fields near the bottom corner. In case $\mathrm{C}$, the kink shadows part of the arc region, and the negative surface charge in the shadow gives the ions an extra kick, causing them all to land on the collector. In case $\mathrm{D}$, the bump is far enough back that the ions are deflected into the arc region, lowering the field there. Having undergone less acceleration, the ions are drawn by transverse fields into the sidewall. Nonetheless, the top part of the sidewall is still shadowed. This sensitivity to the exact shape of the photoresist means that the ion orbits change during the etch and cannot be predicted. Fortunately, the ion flux to the sidewalls is in every case so small compared with that on the collector that it cannot significantly affect the quality of the etch.

\section{Effect of overall geometry}

The conducting surface connected to the trench bottom can extend beyond the trench, and this can affect the electric fields in the trench. To study this, we changed the collector shape. Figure 22 shows three collector geometries. In Fig. 22a, the collector has the same width, 2W, as the trench. In Fig. 22b, the collector has a width of $4 \mathrm{~W}$ or $8 \mathrm{~W}$. In Fig. 22c, the trench extends into a collector of width $4 \mathrm{~W}$. This represents the case of polysilicon etch, the entire dielectric part being photoresist.

1. Change of collector width. When the collector width is increased, the number of ions hitting the sidewall is shown in Table 3 for $A_{\mathrm{R}}=5$, two values of $V_{\mathrm{c}}$, and three collector widths, taken from a total sample of 26,515 ions. The distribution of ions on the sidewall in the $V_{\mathrm{c}}=-26 \mathrm{~V}$ case is shown in Fig. 23. Though the statistics are poor, the trend is for the wider collectors to allow more ions to the sidewalls. The reason is that the equipotential lines near the trench bottom are essentially straight for collector widths greater than $4 \mathrm{~W}$, while for a width of $2 \mathrm{~W}$ the lines are curved, and the corresponding E-field pulls ions away from the wall. 


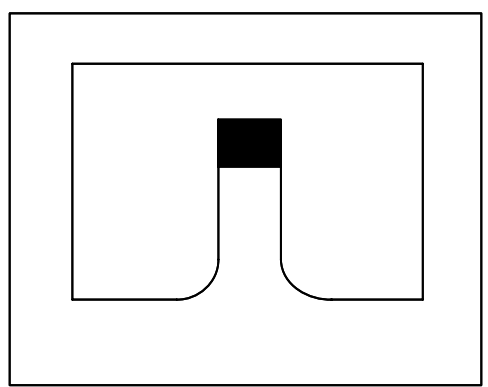

(a)

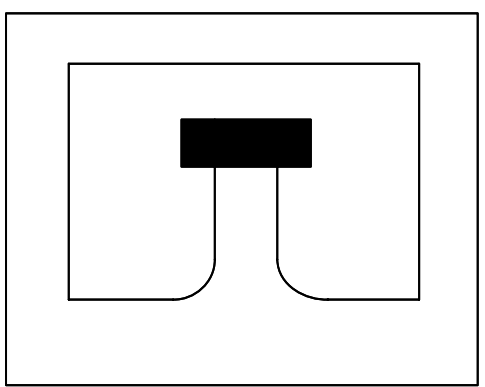

(b)

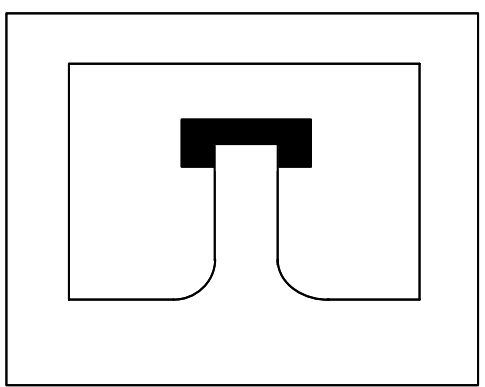

(c)

Fig. 22. Three types of collectors studied.

Table 3: Sidewall ions with wide collectors

\begin{tabular}{|c|c|c|c|}
\hline & $\mathbf{2 W}$ & $\mathbf{4 W}$ & $\mathbf{8 W}$ \\
\hline $\mathbf{- 1 8 V}$ & 141 & 154 & 157 \\
\hline $\mathbf{- 2 6 V}$ & 30 & 35 & 36 \\
\hline
\end{tabular}

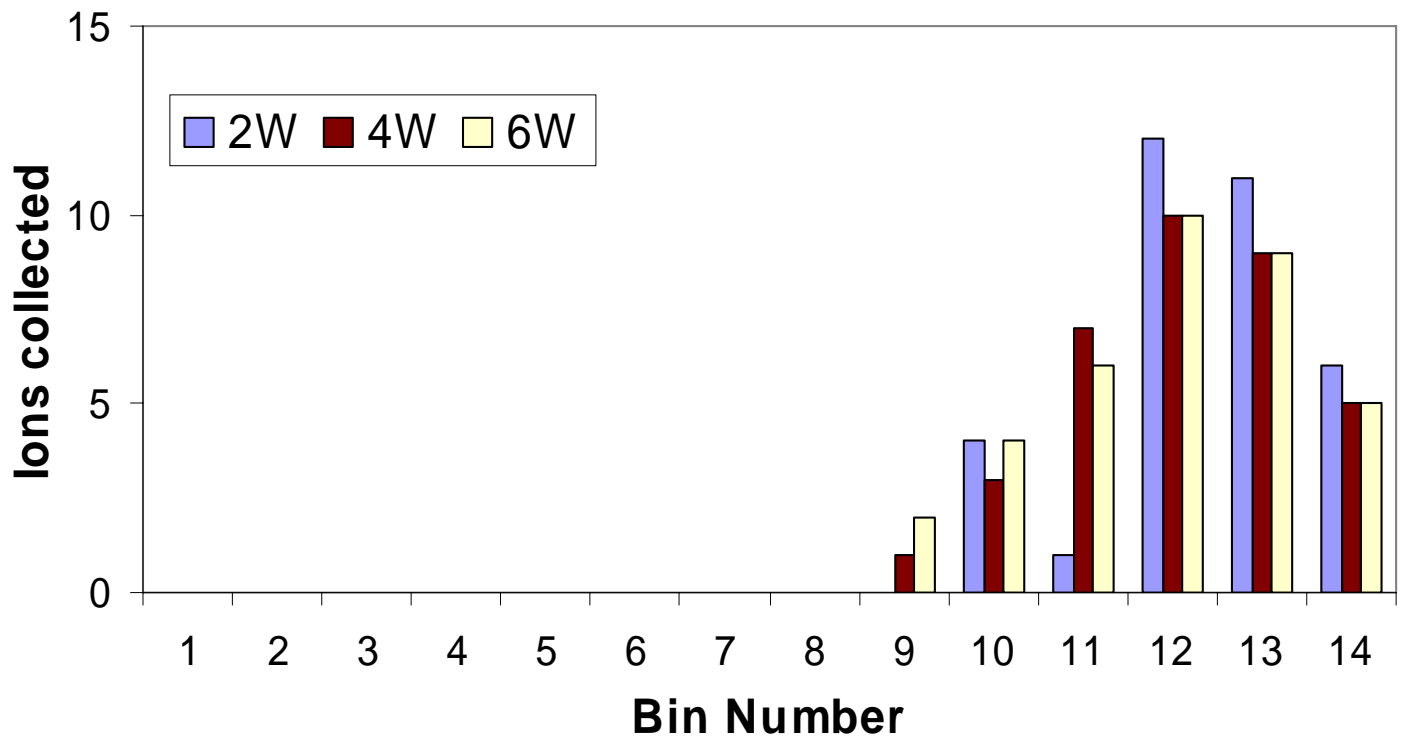

Fig. 23. Distribution of sidewall ions for three collector widths. The entrance is at the left and the collector at the right.

2. Etching into the metal. In the configuration of Fig. 22c, we have examined the cases of $A_{\mathrm{R}}=$ 5 and 7 and $V_{\mathrm{c}}=-26 \mathrm{~V}$ and $-15.5 \mathrm{~V}$, with 2 or 6 bins (out of 14) extending into the metal. The geometry studied is shown in Fig. 24a, and the field pattern in Fig. 24b. Ion orbits near the wall are shown in Fig. 25, including the transition between dielectric and metal walls. From these computations, we observed that a) at low $\left|V_{c}\right|$ ions landed in both the metal and the dielectric parts of the sidewall, b) deeper penetration into the metal results in fewer ions to the dielectric, and c) the same trends occur at $A_{\mathrm{R}}=5$ as at $A_{\mathrm{R}}=7$. 


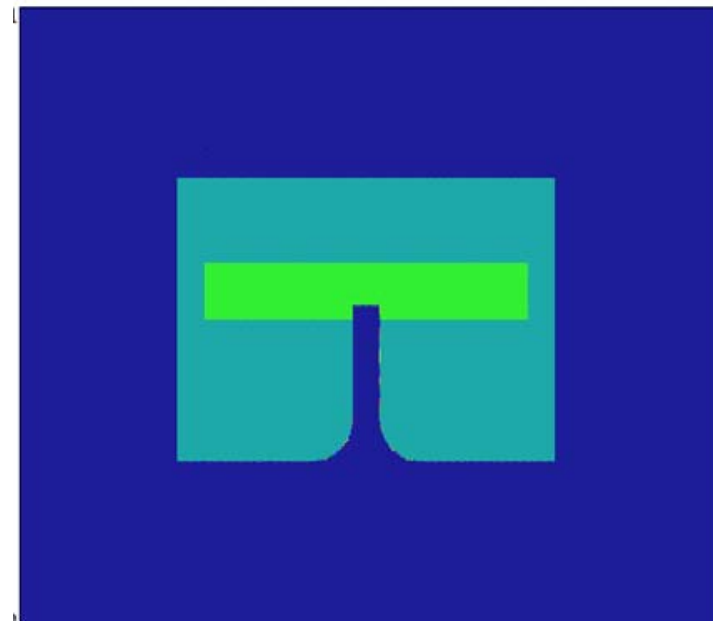

(a)

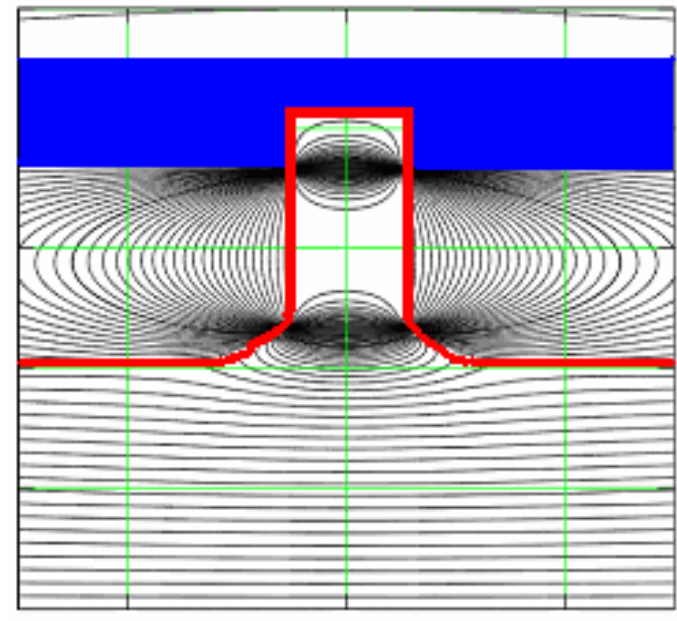

(b)

Fig. 24. Computational region (a) and equipotential lines (b) for trenches extending into the metal.

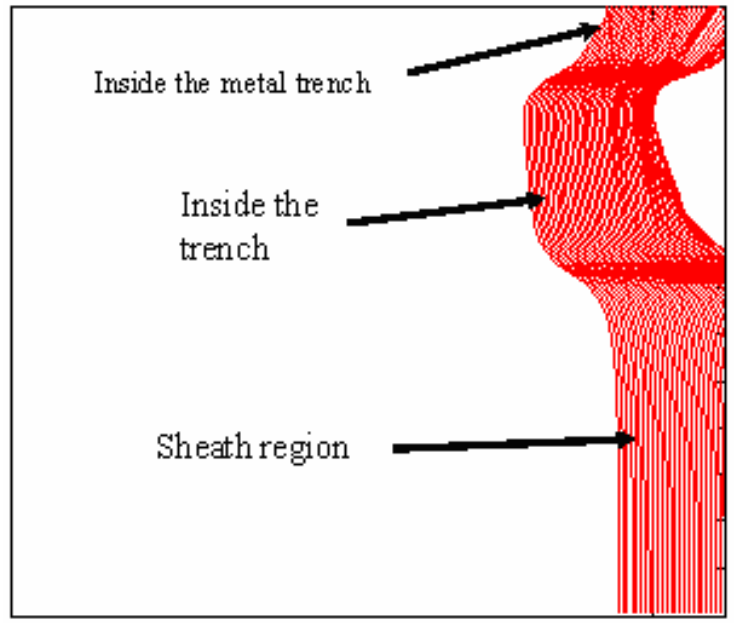

Fig. 25. Ion orbits near the wall for $A_{\mathrm{R}}=5$ and $V_{\mathrm{c}}=-15.5 \mathrm{~V}$.

3. Multiple trenches. To see the effect of neighboring trenches, we considered the double trench configuration shown in Fig. 26a. Since both collectors are biased to the same potential, the ions in each trench are attracted by the field of the collector on the other trench. This results in an asymmetry, seen in the orbits of Fig. 26b and the plot of Fig. 27, such that more ions strike the inner sidewall than the outer one. This asymmetry is shown quantitatively in the histogram of Fig. 28, where it is seen that the collector current is also asymmetric. Compared with an isolated trench, there are more sidewall ions: $2.6 \%$ of 25,000 injected ions vs. $0.5 \%$, or an increase of $2.5 \%$ per trench. Even with this increase, the fraction of all ions entering a trench is still very small, and it decreases with increasing $\left|V_{\mathrm{c}}\right|$. 


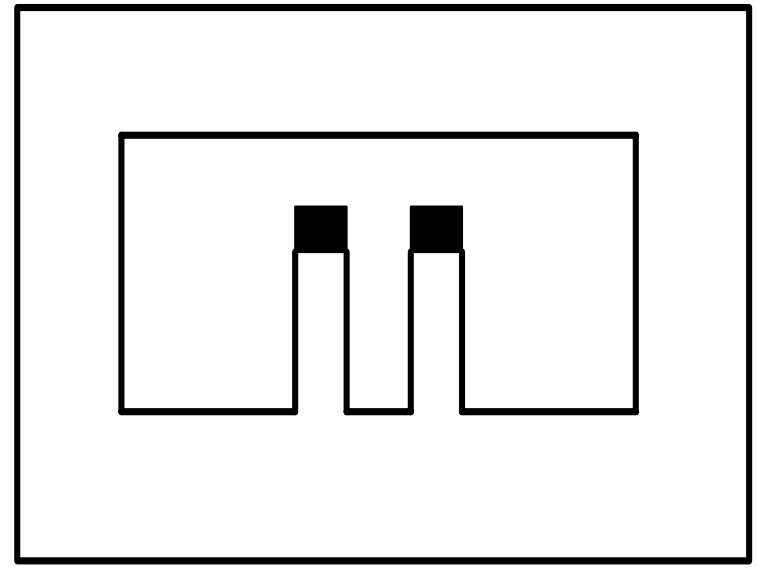

(a)

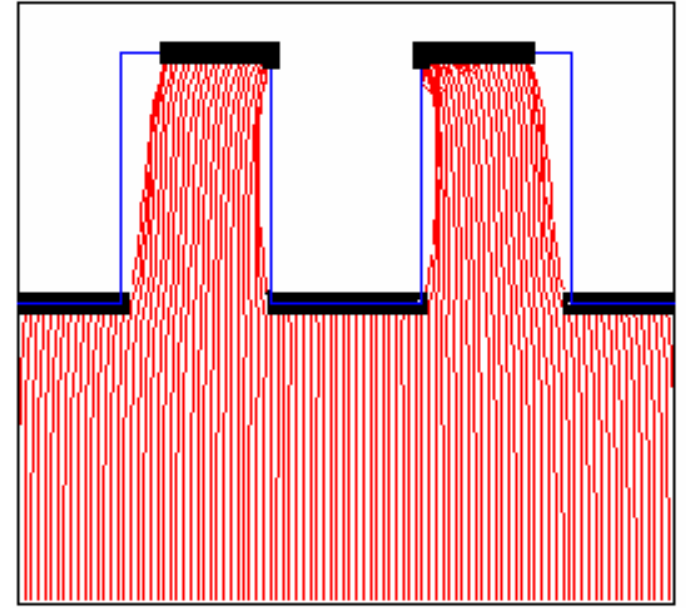

(b)

Fig. 26. (a) A double-trench configuration. (b) Ion orbits in neighboring trenches, with $A_{\mathrm{R}}=5$ and $V_{\mathrm{c}}=-26 \mathrm{~V}$.

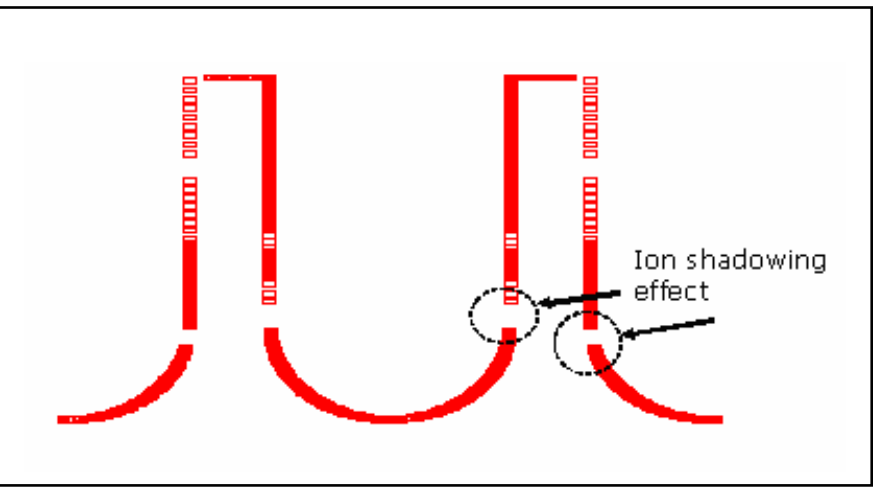

Fig. 27. Ion distribution on sidewalls of a double trench.

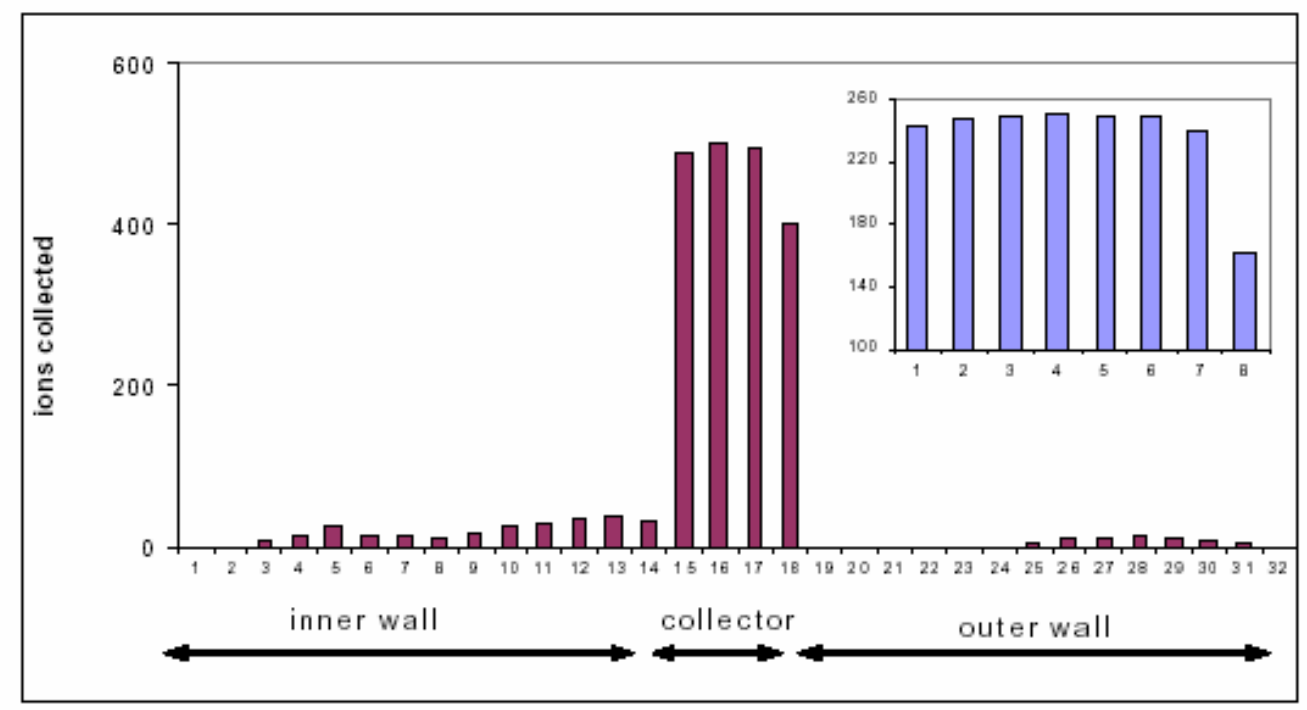

Fig. 28. Distribution of ions collected on the inner and outer walls and on the collector of neighboring trenches. The inset is a detail of the distribution on the collector. 
4. Effect of dielectric constant. In our calculations we have assumed a dielectric constant $\kappa$ of 4 for all insulating materials. To see if a low-k material changes the results, we have compared $\kappa$ $=4$ with the rather extreme value $\kappa=2$ and show results for the case $A_{R}=5, V_{c}=-24 \mathrm{~V}$. The ions suffer small deflections at $\kappa=2$, resulting in a lower percentage of sidewall ions: $0.06 \%$ vs. $0.11 \%$. Their distribution in the sidewall bins is shown in Fig. 29. Since the numbers are so small, this difference does not affect our general conclusions.
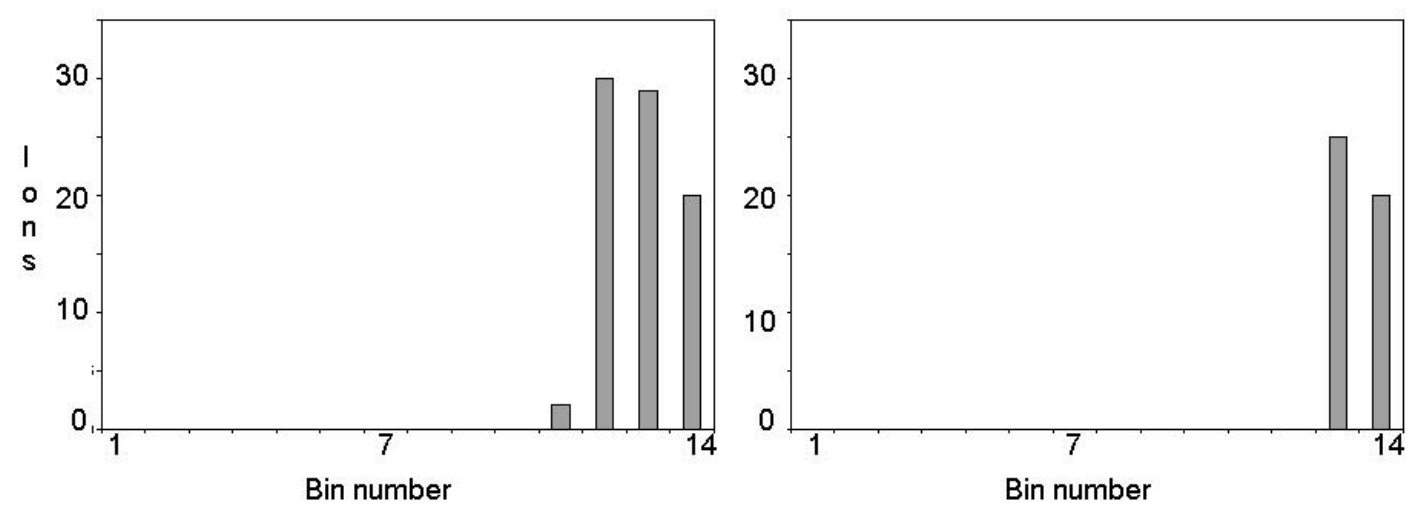

Fig. 29. Distribution of ions along the trench wall for $\kappa=4$ (left) and $\kappa=2$ (right). The collector is at the right.

$$
A_{\mathrm{R}}=5, V_{\mathrm{c}}=-26 \mathrm{~V} \text {. }
$$

\section{LIMIT CYCLES}

The iteration procedure we have used does not always converge. If we plot an output quantity, such as the number of sidewall ions, vs. iteration number, several types of behavior might be expected, as shown in Fig. 30. One hopes for convergence as shown in (a), or at least oscillations damping to the final result, as in (b). Unfortunately, we observe cases in which the result oscillates between two or more values, as in (c) or (d), but at least not as in (e).
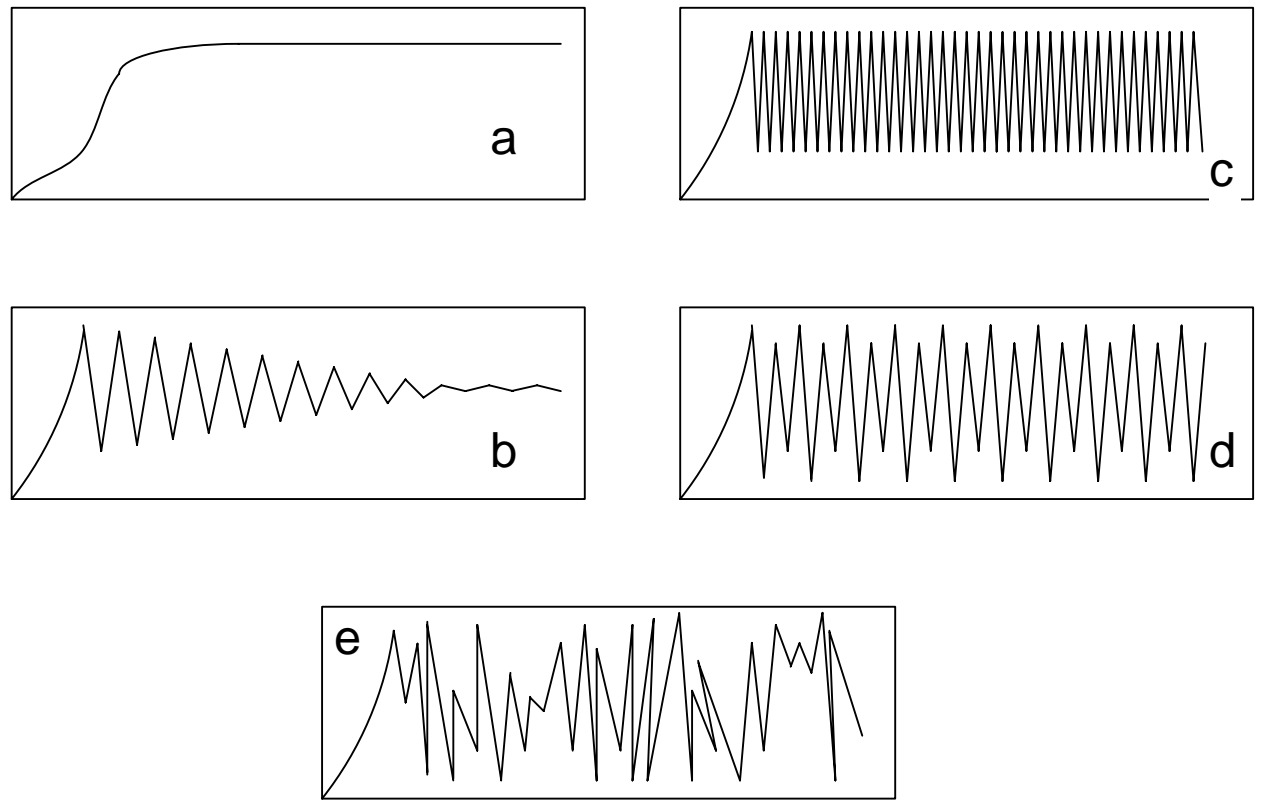

Fig. 30. Types of convergence: (a) steady approach to result, (b) damped oscillations, (c) 2-limit cycle, (d) 3-limit cycle, (e) non-repetitive chaos. 
Figure 31 shows a typical evolution of the profiles of sidewall ions with iteration number. In the zeroth iteration, all the bins have the normal floating potential, Eq. (11); and the ion orbits calculated in this field give the first ion distribution. The potential on each bin is then calculated from Eq. (14), and a smooth curve is passed through $V\left(y_{\mathrm{j}}\right)$ to generate the potentials for the next iteration. Note that the most negative voltage is about $-40 \mathrm{~V}$, corresponding to one ion landing on that bin. This is to avoid the divergence of Eq. (14) when no ion is collected. The results are not sensitive to this arbitrary cutoff. In this example, the ion distribution converges after about

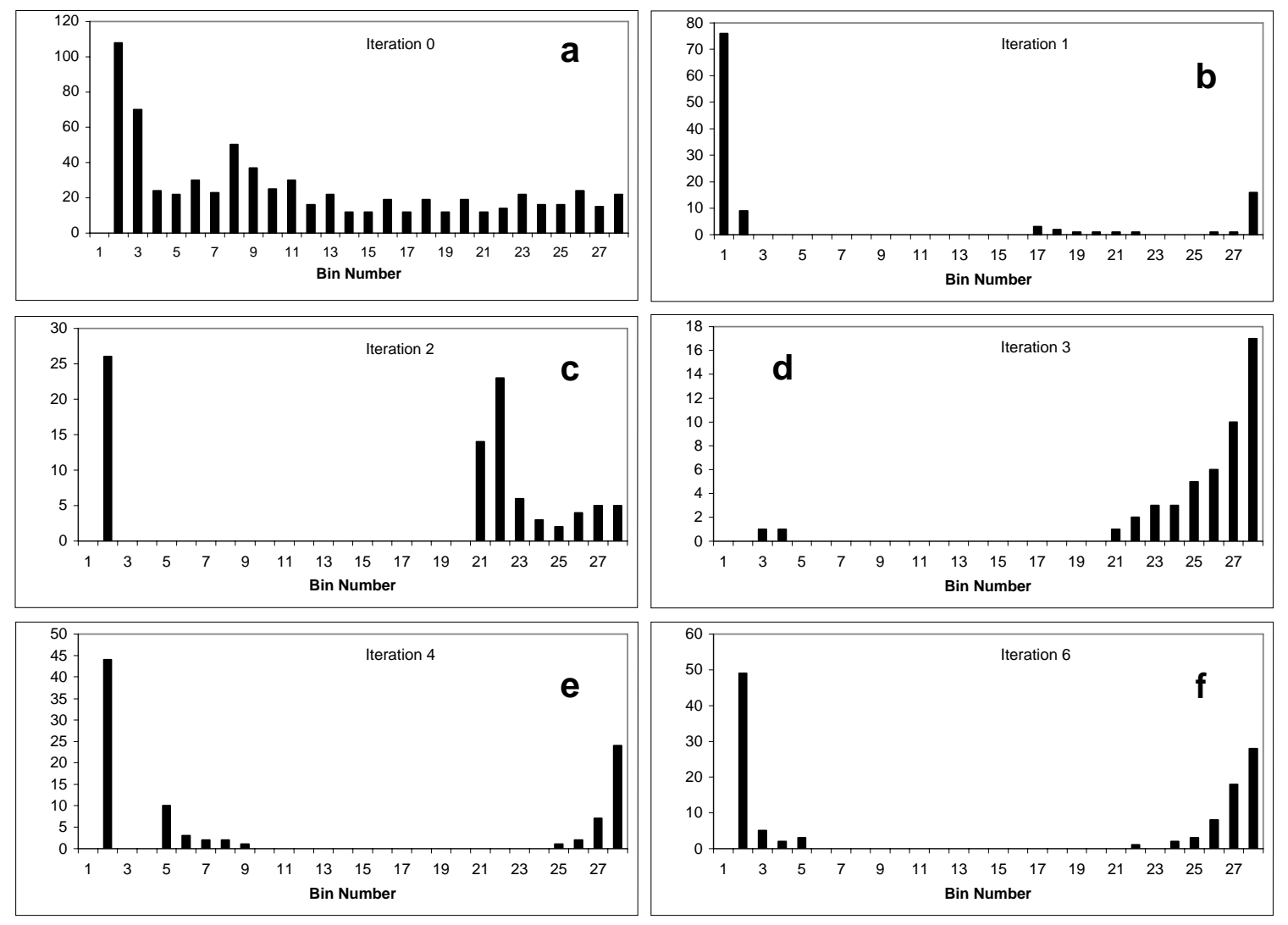

Fig. 31. Progression of sidewall ion distribution with interation number.

seven iterations. In other cases, the ion distribution oscillates between two or among three values, as shown in Fig. 32 for $A_{R}=5, V_{c}=-18 \mathrm{~V}$. In this case, there are three values to which the iteration converges, with a periodicity of three iterations. Figure 32 also shows that although the number of sidewall ions oscillates, the total number entering the trench is constant, the other ions having been directed into the collector. The number of ions falling onto the arc section remains constant. Figure 33 shows the distributions for the three states among which the system oscillates. From this it is seen that these states correspond to different populations of ions in the last few bins near the collector, and the potential at these bins affects the trajectories in the next iteration.

This limit cycle behavior occurs only at low values of $A_{\mathrm{R}}$ and $\left|V_{\mathrm{c}}\right|$, since at high values there are no ions reaching the sidewalls. Table 4 shows the values of $V_{\mathrm{c}}$ for which limit cycles have been observed at each $A_{R}$. From this one can deduce a boundary between the stable region and the unstable region with limit cycles. This is shown in Fig. 34, in which the boundary is fit with an exponential function. 


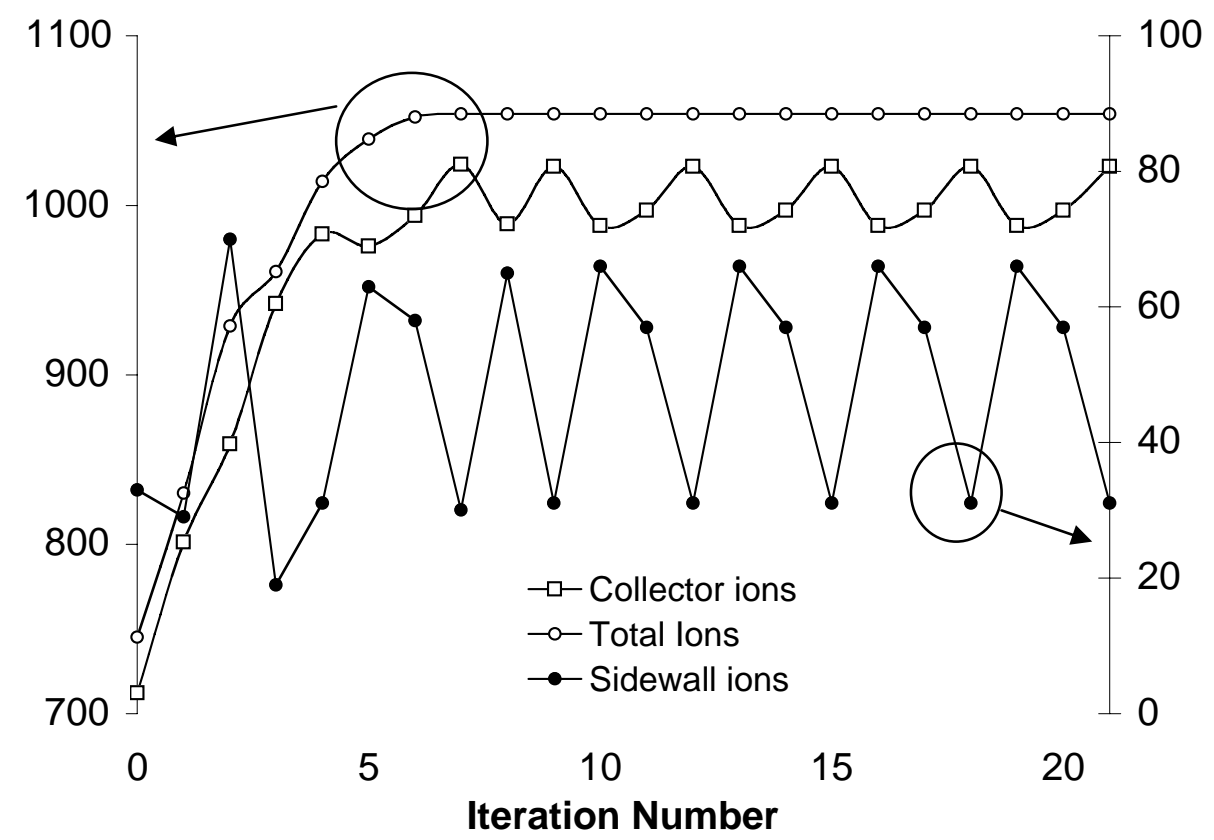

Fig. 32. Variation of sidewall ions $(\bullet)$, collector ions $(\square)$, and total trench ions $(O)$ with iteration number.
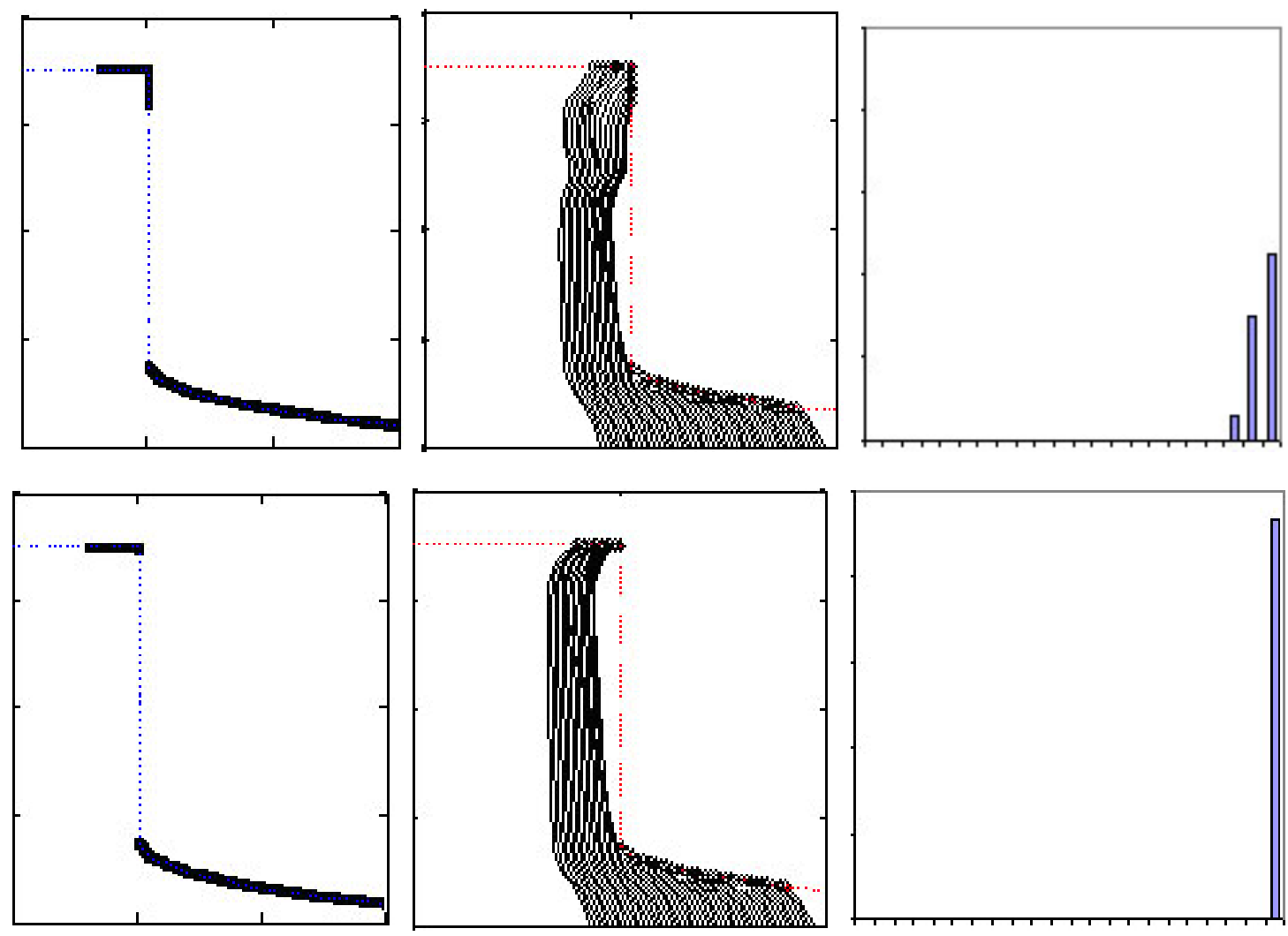

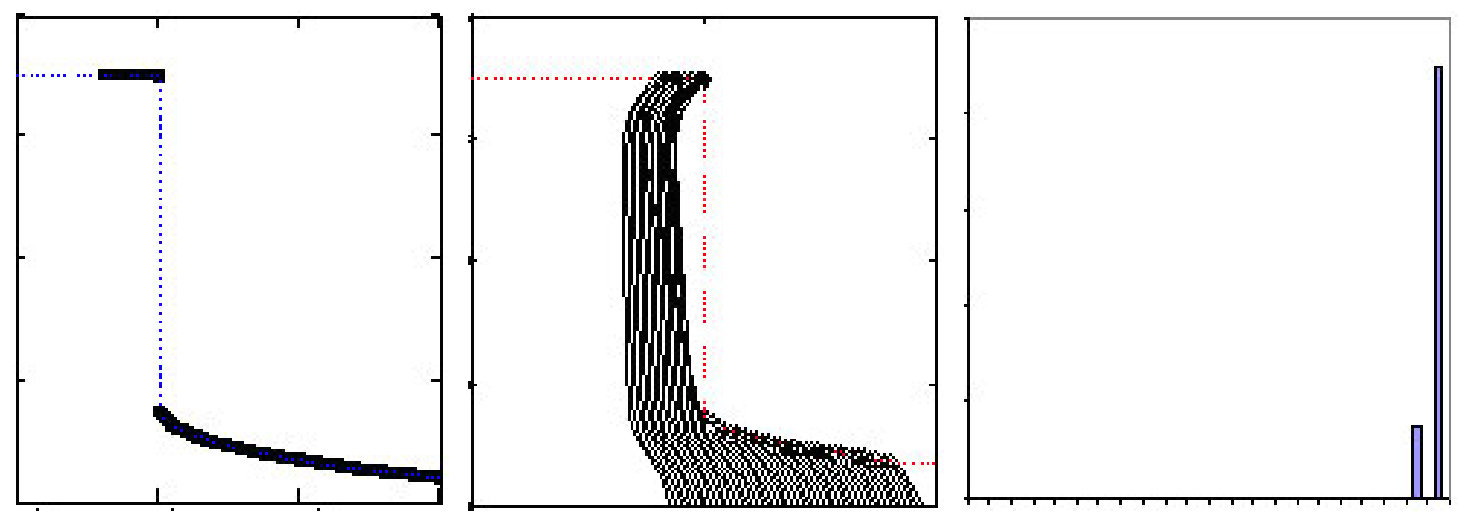

Fig. 33. Ion distributions, ion orbits, and sidewall ions for the three states in a 3-limit cycle, for $A_{R}=5, V_{c}=-18 V$.

Table 4: Limit cycle cases

\begin{tabular}{|c|c|}
\hline $\boldsymbol{A}_{\mathbf{R}}$ & $\boldsymbol{V}_{\mathbf{c}}$ \\
\hline 7 & -15.5 \\
& -18 \\
\hline \multirow{2}{*}{5} & -15.5 \\
& -18 \\
& -22 \\
\hline & -15.5 \\
3 & -18 \\
& -22 \\
& -26 \\
\hline
\end{tabular}

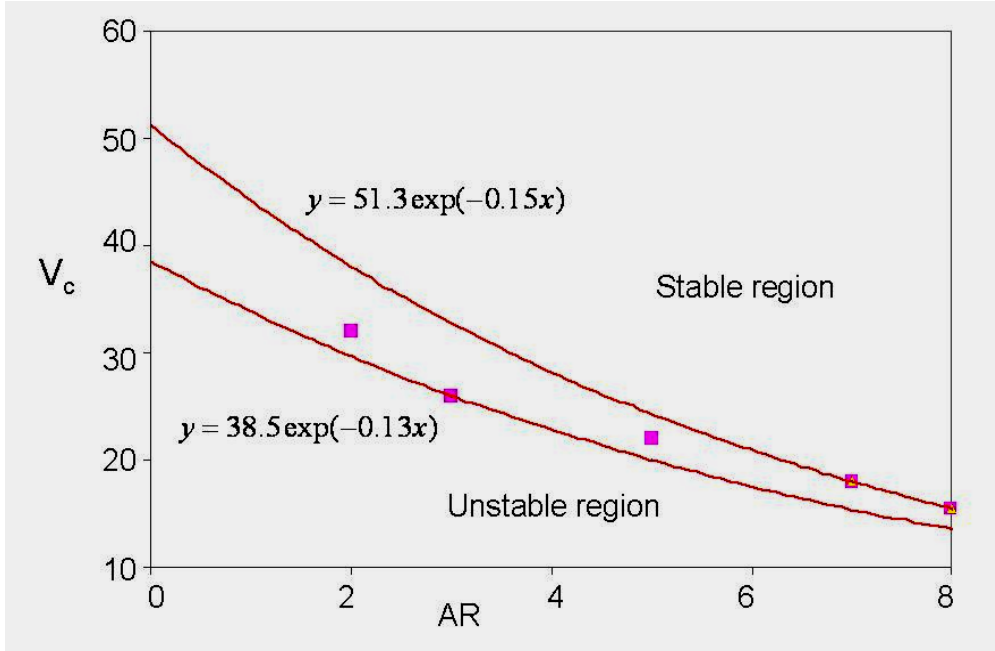

Fig. 34. Limiting cases for iteration convergence, showing a relation between $A_{\mathrm{R}}$ and $V_{\mathrm{c}}$ for the boundary between stable and unstable regions.

The obvious reason for non-convergence is that the computation is too coarse, and we have made several tests to show that this is a persistent effect. First, we quadrupled the number of injected ions. This simply quadrupled the number of ions collected in each bin and did not 
change their distribution or the potentials. This was expected, since our formulation uses only the ratio of $n_{\mathrm{e}}$ to $n_{\mathrm{i}}$ and not the absolute charge. The ion count is only slightly affected by the closer spacing of the orbits. Second, we doubled the size of the sidewall bins, reducing the number from 14 to 7 . Figure 35 shows that the sidewall distribution is not changed, and that limit cycles still occur. However, the smaller bin size leads to faster approach to the oscillating behavior.
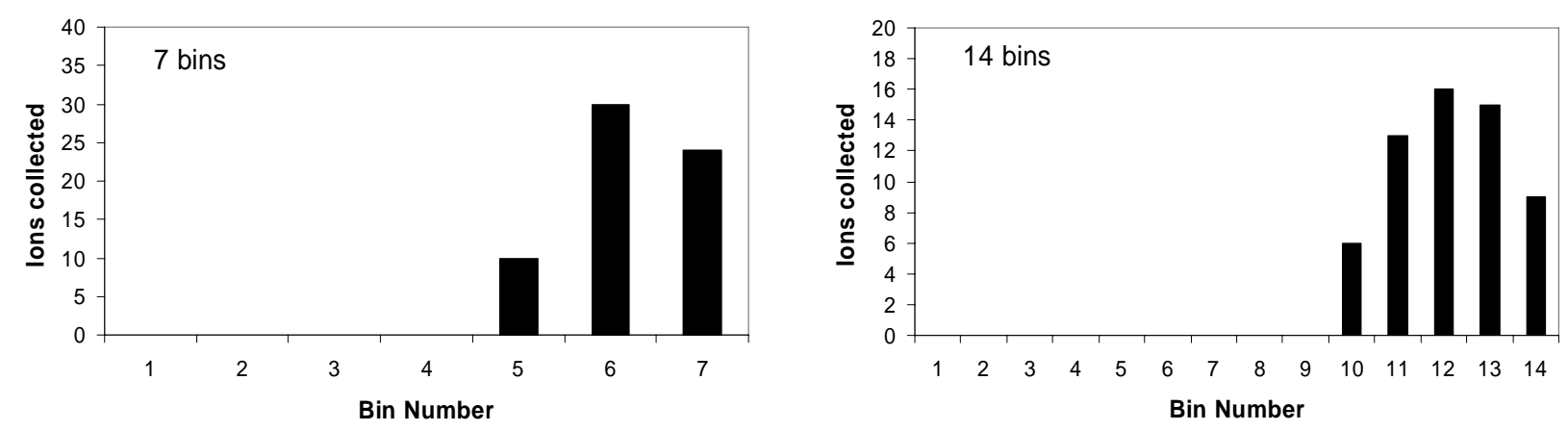

Fig. 35. Effect of bin size on sidewall ion distribution. $A_{\mathrm{R}}=5, V_{\mathrm{c}}=-18 \mathrm{~V}$.

Third, we smoothed the ion sidewall distribution algebraically before computing the potential distribution, using the formula

$$
<N_{j}>=\frac{1}{5} N_{j-1}+\frac{3}{5} N_{j}+\frac{1}{5} N_{j-1} .
$$

This is equivalent to introducing a surface conductivity along the sidewalls, which is as much as can be done since this conductivity is unknown and probably not constant. The limit cycles still persist, though it takes more iterations to reach them. There is a small change in the number of sidewall ions, but they are all near the trench corner. A change in the weighting factors in Eq. (15) does not remove the limit cycles.

The lack of convergence can be traced to ion shading at the trench entrance. Typically, the strong fields there deflect the ions so that they land on only a few bins at the far end of the trench. When the aspect ratio is small, the charges there can affect the E-fields to, say, repel ions from the sidewall so that fewer will land on the crucial bins. This in turn diminishes the repulsive force and allows more ions to land there in the next iteration. Limit cycles of small amplitude will always occur as long as the bins have finite width, but they can be made negligibly small by greatly increasing the number of sidewall bins and also decreasing the electromagnetic mesh size near the corners. When limit cycles occurred in the present work, we simply took the average of the repeating states, without any effect on our general conclusions. Since this work was first reported ${ }^{5,6}$, limit cycles have also been encountered in a much larger computation $^{7}$; there, convergence was artificially obtained by averaging over previous iterations.

\section{SUMMARY AND CONCLUSIONS}

By reducing the problem to a simple dimensionless form, universal curves were obtained for ion trajectories in etched trenches, self-consistently with the sidewall potentials. Reflection of ions from the walls was ignored. Results depend on only two parameters: the aspect ratio $A_{R}$, and the potential $V_{\mathrm{c}}$ at the trench bottom. Principal findings are as follows: 
1. Electric fields external to the trench cause the ions to enter the trench at an angle. This causes an ion shading effect which protects the top part of the trench, and sometimes all of it, from ion bombardment.

2. Ion orbits are determined mainly by the strong fields at the corners of the entrance; they then coast through the trench with little additional deflection.

3. In spite of the strong fields at the entrance, ion orbits are nearly straight and vertical. So few ions strike the sidewall that they cannot cause deformations of the trench profile.

4. The small ion flux to the walls is very sensitive to the exact shape of the photoresist at the top. This will change in an unpredictable way during the etching process.

5. Contrary to intuition, a deep trench with large $A_{\mathrm{R}}$ will have no ions striking the wall.

These observations differ from commonly held conceptions of how the ion flux behaves in reactive ion etching. By examining the variation with $A_{R}$, one can get an idea of the changes in ion behavior as the etch progresses. By varying $V_{\mathrm{c}}$, one can gauge the changes during an rf cycle. The conclusions above are not affected by the convergence difficulties in the iteration process. Finally, the scale invariance of the problem permits a scaled-up experiment, at very low density, in which the computational results can be checked. An attempt at such an experiment is described elsewhere ${ }^{4}$.

\section{ACKNOWLEDGMENTS}

This work was supported by UC Discovery Grants, Applied Materials, Inc., and the National Science Foundation. 


\section{REFERENCES}

a) Electronic mail: tsitsi@lanl.gov

b) Deceased

c) Electronic mail: ffchen@ee.ucla.edu

${ }^{1}$ K. Hashimoto, Jpn. J. Appl. Phys. 33, 6013 (1994).

${ }^{2}$ G.S. Hwang and K.P. Giapis, J. Vac. Soc. Technol. B 15, 70 (1997).

${ }^{3}$ S. Humphries, Field Precision, Inc., TriComp E-Stat

${ }^{4}$ T.G. Madziwa-Nussinov, Thesis, UCLA (2003).

${ }^{5}$ T.G. Madziwa and F.F. Chen, Bull. Amer. Phys. Soc. 45(6), 51 (2000), Gaseous Elec. Conf., Houston, TX, Oct. 2000, unpublished.

${ }^{6}$ T.G. Madziwa, F.F. Chen, and D. Arnush, Ion trajectories in electron shading damage, Amer. Vacuum Soc., Denver, CO (2002), paper PS-TuP22.

${ }^{7}$ A.P. Mahorowala and H.H. Sawin, J. Vac. Sci. Technol. B 20, 1084 (2002). 